\title{
Positivity for special cases of $(q, t)$-Kostka coefficients and standard tableaux statistics
}

\author{
Mike Zabrocki \\ Centre de Recherche Mathématiques, Université \\ de Montréal/LaCIM, Université de Québec à Montréal \\ email: zabrocki@math.ucsd.edu
}

MR Subject Number: 05E10

\begin{abstract}
We present two symmetric function operators $H_{3}^{q t}$ and $H_{4}^{q t}$ that have the property $H_{3}^{q t} H_{\left(2^{a} 1^{b}\right)}[X ; q, t]=H_{\left(32^{a} 1^{b}\right)}[X ; q, t]$ and $H_{4}^{q t} H_{\left(2^{a} 1^{b}\right)}[X ; q, t]=H_{\left(42^{a} 1^{b}\right)}[X ; q, t]$. These operators are generalizations of the analogous operator $H_{2}^{q t}$ and also have expressions in terms of Hall-Littlewood vertex operators. We also discuss statistics, $a_{\mu}(T)$ and $b_{\mu}(T)$, on standard tableaux such that the $q, t$ Kostka polynomials are given by the sum over standard tableaux of shape $\lambda, K_{\lambda \mu}(q, t)=\sum_{T} t^{a_{\mu}(T)} q^{b_{\mu}(T)}$ for the case when when $\mu$ is two columns or of the form $\left(32^{a} 1^{b}\right)$ or $\left(42^{a} 1^{b}\right)$. This provides proof of the positivity of the $(q, t)$-Kostka coefficients in the previously unknown cases of $K_{\lambda\left(32^{a} 1^{b}\right)}(q, t)$ and $K_{\lambda\left(42^{a} 1^{b}\right)}(q, t)$. The vertex operator formulas are used to give formulas for generating functions for classes of standard tableaux that generalize the case when $\mu$ is two columns.
\end{abstract}

\section{Introduction and Notation}

A partition $\lambda$ is a weakly decreasing sequence of non-negative integers with $\lambda_{1} \geq \lambda_{2} \geq \ldots \geq$ $\lambda_{k} \geq 0$. The length $l(\lambda)$ of the partition is the largest $i$ such that $\lambda_{i}>0$. The partition $\lambda$ is a partition of $n$ if $\lambda_{1}+\lambda_{2}+\cdots+\lambda_{l(\lambda)}=n$. Young diagrams will be drawn using the French notation with the longest row on the bottom and will be identified with the partition itself by referring to a partition as a collection of cells. 
For every partition $\lambda$ there is a corresponding conjugate partition denoted by $\lambda^{\prime}$ where $\lambda_{i}^{\prime}=$ the number of cells in the $i^{\text {th }}$ column of $\lambda$. The arm of a cell $s$ in $\mu$ will be denoted by $a_{\mu}(s)$ and is the number of cells that lie to the east of $s$ in $\mu$. The leg, $l_{\mu}(s)$, is the number of cells in $\mu$ that are strictly north.

A skew partition is denoted by $\lambda / \mu$, where it is assumed that $\mu \subseteq \lambda$, and represents the cells that are in $\lambda$ but are not in $\mu$. A skew partition $\lambda / \mu$ is said to be a horizontal strip if there is at most one cell in each column. Denote the class of horizontal strips of size $k$ by $\mathcal{H}_{k}$ so that the notation $\lambda / \mu \in \mathcal{H}_{k}$ means that $\lambda / \mu$ is a horizontal strip with $k$ cells. Similarly, the class of vertical strips (skew partitions with at most one cell in each row) will be denoted by $\mathcal{V}_{k}$.

If $\lambda$ is a partition, then let $\lambda^{r}$ denote the partition with the first row removed, that is $\lambda^{r}=\left(\lambda_{2}, \lambda_{3}, \ldots, \lambda_{l(\lambda)}\right)$. Let $\lambda^{c}$ denote the partition with the first column removed, so that $\lambda^{c}=\left(\lambda_{1}-1, \lambda_{2}-1, \ldots, \lambda_{l(\lambda)}-1\right)$. This allows us to define the border of a partition $\mu$ to be the skew partition $\mu / \mu^{r c}$. Define the $k$-snake of a partition $\mu$ to be the $k$ bottom most right hand cells of the border of $\mu$ (the choice of the word "snake" is supposed to suggest the cells that slink from the bottom of the partition up along the right hand edge). We use the symbol $h t_{k}(\mu)$ to denote the height of the $k$-snake. The symbol $\left.\mu\right\rfloor_{k}$ will be used to represent a partition with the $k$-snake removed with the understanding that if removing the $k$-snake does not leave a partition that this symbol is undefined.

\section{Examples}

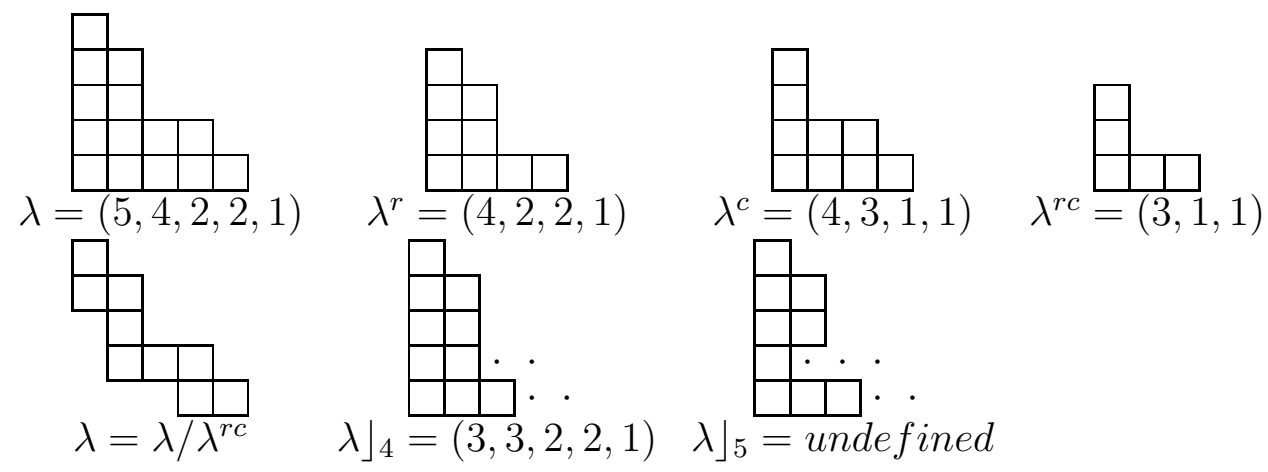

If the shape of $\rho=\lambda\rfloor_{k}$ is given and the height of the $k$-snake is specified then $\lambda$ can be recovered ( $\lambda$ is determined from $\rho$ by adding a $k$-snake of height $h$ ). This is because $\lambda=\left(\rho_{h}+k-h+1, \rho_{1}+1, \rho_{2}+1, \ldots, \rho_{h-1}+1, \rho_{h+1}, \rho_{h+2}, \ldots, \rho_{l(\rho)}\right)$. This will be a partition as long as $k$ is sufficiently large. 
We will consider the ring of symmetric functions in an infinite number of variables as a subring of $\mathbb{Q}\left[x_{1}, x_{2}, \ldots\right]$. A more precise construction of this ring can be found in [19] section I.2 (and roughly, the notation of this reference will be followed).

The Macdonald integral basis [19] for the symmetric functions are defined by the following two conditions

$$
\begin{aligned}
& \text { a) } J_{\lambda}=\prod_{s \in \lambda}\left(1-q^{a_{\lambda}(s)} t^{l_{\lambda}(s)+1}\right) s_{\lambda}+\sum_{\mu<\lambda} s_{\mu} c_{\mu \lambda}(q, t) \\
& (s \in \lambda \text { means run over all cells } s \text { in } \lambda) \\
& \text { b) }\left\langle J_{\lambda}, J_{\mu}\right\rangle_{q t}=0 \text { for } \lambda \neq \mu
\end{aligned}
$$

where $\langle,\rangle_{q t}$ denotes the scalar product of symmetric functions defined on the power symmetric functions by $\left\langle p_{\lambda}, p_{\mu}\right\rangle_{q t}=\delta_{\lambda \mu} z_{\lambda} \prod_{k} \frac{1-q^{\lambda} k}{1-t^{\lambda} k}\left(z_{\lambda}\right.$ is the size of the stablizer of the permutations of cycle structure $\lambda$ and $\delta_{x y}=1$ if $x=y$ and 0 otherwise). The coefficients $c_{\mu \lambda}(q, t)$ are determined by these two conditions and are rational functions in $q$ and $t$.

Also define an additional scalar product $\left\langle p_{\lambda}, p_{\mu}\right\rangle_{t}=\delta_{\lambda \mu} z_{\lambda} \prod_{k} \frac{1}{1-t^{\lambda_{k}}}$. The $(q, t)$-Kostka coefficients are then given by the expression $K_{\lambda \mu}(q, t)=\left\langle J_{\mu}[X ; q, t], s_{\lambda}[X]\right\rangle_{t}$.

We will also refer to the basis $H_{\mu}[X ; q, t]=\sum_{\lambda} K_{\lambda \mu}(q, t) s_{\lambda}[X]$ that is of interest in this paper as Macdonald symmetric functions. The $H$ basis is related to the $J$ basis by a plethystic transformation. Define the basis $H_{\mu}[X ; t]=H_{\mu}[X ; 0, t]$ as Hall-Littlewood symmetric functions. The $H_{\mu}[X ; t]$ are a transformed version of the Hall-Littlewood polynomials defined in [19] and are analogous to the $H_{\mu}[X ; q, t]$.

We will use the notation of $f^{\perp}$ to denote the adjoint to multiplication for a symmetric function $f$ with respect to the standard inner product. Therefore $\left\langle f^{\perp} g, h\right\rangle=\langle g, f h\rangle$. Note that $h_{k}^{\perp}$ and $e_{k}^{\perp}$ act on the Schur function basis with the formulas

$$
e_{k}^{\perp} s_{\mu}=\sum_{\mu / \lambda \in \mathcal{V}_{k}} s_{\lambda} \quad h_{k}^{\perp} s_{\mu}=\sum_{\mu / \lambda \in \mathcal{H}_{k}} s_{\lambda}
$$

A Schur symmetric function vertex operator is due to Bernstein [19, p. 95-6] and is given by the formula

$$
S_{m}=\sum_{k \geq 0}(-1)^{k} h_{m+k} e_{k}^{\perp}
$$

It has the property that for $m \geq \mu_{1}, S_{m} s_{\mu}[X]=s_{(m, \mu)}[X]$. 
There is a Hall-Littlewood symmetric function vertex operator

$$
H_{m}^{t}=\sum_{i, j \geq 0} t^{j}(-1)^{i} h_{m+i+j}[X] e_{i}^{\perp} h_{j}^{\perp}=\sum_{k \geq 0} t^{k} S_{m+k} h_{k}^{\perp}
$$

with the property that for $m \geq \mu_{1}$ that $H_{m}^{t} H_{\mu}[X ; t]=H_{(m, \mu)}[X ; t]$ which is due to Jing ([9], [5, [19]). This symmetric function operator can be used to prove the existence of statistics on column strict tableaux such that $H_{\mu}[X ; t]=\sum_{T} t^{c(T)} s_{\lambda(T)}[X]$. The action of this operator on the Schur function basis can be expressed as follows [23.

Proposition 1.1 Let $\lambda$ be a partition of $n$, let $m$ be a non-negative integer and let $k$ be any non-negative integer such that $m+k \geq \lambda_{1}$, then

$$
H_{m} s_{\lambda}[X]=\sum_{\mu / \lambda \in \mathcal{H}_{m+k}}(-1)^{h t_{k}(\mu)-1} t^{\left|\lambda / \mu^{r}\right|} s_{\mu]_{k}}[X]
$$

with the understanding that if $\mu\rfloor_{k}$ is not defined then there is no contribution from that term.

The object of this research is to find an analogous operator to $H_{m}^{t}$ for the Macdonald symmetric functions $H_{\mu}[X ; q, t], H_{m}^{q t}$, and use it to derive statistics on standard tableaux that count the terms in $H_{\mu}[X ; q, t]$.

Introduce the notation for the operator that acts on symmetric functions of homogeneous degree $n$ with the formula

$$
\bar{H}_{m}^{t}=\omega H_{m}^{\frac{1}{t}} \omega R^{t}=\sum_{i, j \geq 0} t^{n-j}(-1)^{i} e_{m+i+j}[X] h_{i}^{\perp} e_{j}^{\perp}
$$

where $R^{t}$ is an operator with the property that $R^{t} P[X]=t^{n} P[X]$ for $P[X]$ a homogeneous polynomial of degree $n$. In 22] we show that

Theorem 1.2 The operator

$$
H_{2}^{q t}=H_{2}^{t}+q \bar{H}_{2}^{t}
$$

has the property that $H_{2}^{q t} H_{\left(2^{a} 1^{b}\right)}[X ; q, t]=H_{\left(2^{a+1} 1^{b}\right)}[X ; q, t]$.

In addition we used this operator to show that there exists statistics on standard tableaux $a_{\mu}(T)$ and $b_{\mu}(T)$ for $\mu=\left(2^{a} 1^{b}\right)$ such that

$$
H_{\mu}[X ; q, t]=\sum_{T} q^{b_{\mu}(T)} t^{a_{\mu}(T)} s_{\lambda(T)}[X]
$$


where the sum is over standard tableaux of size $2 a+b$.

This case was already considered by Susanna Fischel in [3] where it was shown that the $q, t$-Kostka coefficients are a sum over a subclass of rigged configurations [11, [12. There exists a bijection between rigged configurations and standard tableaux, but the isomorphism between the two sets of objects is not trivial [8, 13], [14. This case was also considered in [16] using a similar approach but with a different vertex operator for the Macdonald polynomials.

The problem becomes more difficult when $m=3$ and we will present here a formula for the vertex operator that adds a row of size 3, but only works when it acts on the Macdonald polynomials indexed by less than or equal two columns.

Theorem 1.3 The operator

$$
\begin{aligned}
H_{3}^{q t} & =H_{3}^{t}+\left(e_{1}[X] H_{2}^{t}-H_{3}^{t}\right) q+\left(e_{1}[X] \bar{H}_{2}^{t}-\bar{H}_{3}^{t}\right) q^{2}+\bar{H}_{3}^{t} q^{3} \\
& =(1-q)\left(H_{3}^{t}-q^{2} \bar{H}_{3}^{t}\right)+q e_{1}[X] H_{2}^{q t}
\end{aligned}
$$

has the property that $H_{3}^{q t} H_{\left(2^{a} 1^{b}\right)}[X ; q, t]=H_{\left(32^{a} 1^{b}\right)}[X ; q, t]$.

We will also present an operator that adds a row of size 4 but again has the property that it only works when it acts on Macdonald polynomials indexed by less than or equal two columns.

Theorem 1.4 The operator

$$
\begin{aligned}
H_{4}^{q t}= & H_{4}^{t}+\left(h_{1}[X] H_{3}^{t}-H_{4}^{t}\right) q+\left(h_{2}[X] H_{2}^{t}-H_{4}^{t}\right) q^{2}+\left(e_{2}[X] H_{2}^{t}-e_{1} H_{3}^{t}+H_{4}^{t}\right) q^{3} \\
& +\left(h_{2}[X] \bar{H}_{2}^{t}-h_{1} \bar{H}_{3}^{t}+\bar{H}_{4}^{t}\right) q^{3}+\left(e_{2}[X] \bar{H}_{2}^{t}-\bar{H}_{4}^{t}\right) q^{4}+\left(e_{1}[X] \bar{H}_{3}^{t}-\bar{H}_{4}^{t}\right) q^{5}+\bar{H}_{4}^{t} q^{6} \\
= & (1-q)\left(1-q^{2}\right)\left(H_{4}^{t}+q^{3} \bar{H}_{4}^{t}\right)-q\left(1-q^{2}\right) e_{1}\left(H_{3}^{t}-q^{2} \bar{H}_{3}^{t}\right)+q^{2}\left(h_{2}+q e_{2}\right)\left(H_{2}^{t}+q \bar{H}_{2}^{t}\right) \\
= & (1-q)\left(1-q^{2}\right)\left(H_{4}^{t}+q^{3} \bar{H}_{4}^{t}\right)+q(1+q) e_{1} H_{3}^{q t}-q^{2}\left(e_{2}+q h_{2}\right) H_{2}^{q t}
\end{aligned}
$$

has the property that $H_{4}^{q t} H_{\left(2^{a} 1^{b}\right)}[X ; q, t]=H_{\left(42^{a} 1^{b}\right)}[X ; q, t]$.

With the three operators $H_{2}^{q t}, H_{3}^{q t}$ and $H_{4}^{q t}$ acting on $H_{1^{b}}[X ; q, t]$ all of the Macdonald polynomials up to $n=8$ except $(3,3,2)$ are quickly computable with a computer algebra package such as Maple with John Stembridge's 'SF' package or Sébastien Veigneau's package 'ACE.'

These two formulas were arrived at by mix of chance, educated guessing, and computer experimentation. The proofs of these operators are not very elegant, but are also not very difficult to follow and only involve repeated applications of the Macdonald Pieri formula [19]. 
They are nice because they are expressed in terms of the Hall-Littlewood vertex operators and the action of these operators is well understood [23. If a formula for the general Macdonald vertex operator of this sort exists, it will be of the same flavor as these but it will generalize the Hall-Littlewood vertex rather than have an expression in terms of it.

In this paper, we will present statistics on standard tableaux that will show that the Macdonald polynomials of the form $H_{\left(32^{a} 1^{b}\right)}[X ; q, t]$ and $H_{\left(42^{a} 1^{b}\right)}[X ; q, t]$, when expanded in terms of Schur symmetric functions, have coefficients that are polynomials in $q$ and $t$ with non-negative integer coefficients (symmetric functions with this property will be called Schur positive).

\section{Formulas for $H_{\left(32^{a} 1^{b}\right)}[X ; q, t]$ and $H_{\left(42^{a} 1^{b}\right)}[X ; q, t]$}

An expansion for the Macdonald polynomials $H_{\left(2^{a} 1^{b}\right)}[X ; q, t]$ in terms of the Hall-Littlewood symmetric functions was given in [20. The coefficients there are factorable and of a nice form. Theorem 1.1 in [20] is the following result

$$
\begin{aligned}
H_{\left(2^{a} 1^{b}\right)}[X ; q, t] & =\sum_{i=0}^{a} q^{a-i}\left(q t^{a+b-i+1} ; t\right)_{i} \frac{(t ; t)_{a}}{(t ; t)_{i}(t ; t)_{a-i}} H_{\left(2^{i} 1^{b+2 a-2 i}\right)}[X ; t] \\
& =\sum_{i=0}^{a} c_{i}^{(a, b)} H_{\left(2^{i} 1^{b+2 a-2 i}\right)}[X ; t]
\end{aligned}
$$

where $(a ; x)_{k}=(1-a)(1-a x) \cdots\left(1-a x^{k-1}\right)$.

Using a translation of the Pieri formula for Macdonald polynomials 19, p. 340, eq. $6.24(\mathrm{iv})]$ onto the $H_{\mu}[X ; q, t]$ basis and that $\mu$ is two columns wide, we can say that

$$
e_{1}[X] H_{\left(2^{a+1} 1^{b}\right)}[X ; q, t]=A H_{\left(32^{a} 1^{b}\right)}[X ; q, t]+B H_{\left(2^{a+2} 1^{b-1}\right)}[X ; q, t]+C H_{\left(2^{a+1} 1^{b+1}\right)}[X ; q, t]
$$

where

$$
\begin{aligned}
& A=\frac{\left(1-t^{a+1}\right)\left(1-q t^{a+b+1}\right)}{\left(1-q^{2} t^{a+b+1}\right)\left(1-q t^{a+1}\right)} \\
& B=\frac{\left(1-t^{b}\right)(1-q)}{\left(1-q t^{b}\right)\left(1-q t^{a+1}\right)} \\
& C=\frac{(1-q)\left(1-q^{2} t^{b}\right)}{\left(1-q t^{b}\right)\left(1-q^{2} t^{a+b+1}\right)}
\end{aligned}
$$


Rearranging this formula to solve for $H_{\left(32^{a} 1^{b}\right)}[X ; q, t]$ gives

$$
H_{\left(32^{a} 1^{b}\right)}[X ; q, t]=A^{\prime} e_{1}[X] H_{\left(2^{a} 1^{b}\right)}[X ; q, t]-B^{\prime} H_{\left(2^{a+2} 1^{b-1}\right)}[X ; q, t]-C^{\prime} H_{\left(2^{a+1} 1^{b+1}\right)}[X ; q, t]
$$

where

$$
\begin{aligned}
A^{\prime} & =\frac{\left(1-q^{2} t^{a+b+1}\right)\left(1-q t^{a+1}\right)}{\left(1-t^{a+1}\right)\left(1-q t^{a+b+1}\right)} \\
B^{\prime} & =\frac{\left(1-q^{2} t^{a+b+1}\right)\left(1-t^{b}\right)(1-q)}{\left(1-t^{a+1}\right)\left(1-q t^{b}\right)\left(1-q t^{a+b+1}\right)} \\
C^{\prime} & =\frac{\left(1-q^{2} t^{b}\right)\left(1-q t^{a+1}\right)(1-q)}{\left(1-t^{a+1}\right)\left(1-q t^{b}\right)\left(1-q t^{a+b+1}\right)}
\end{aligned}
$$

The last two terms on the right hand side of equation (2.6) can be expanded in terms of Hall-Littlewood symmetric functions using (2.1). The first term on the right hand side of the equation can be expanded in terms of Hall-Littlewood symmetric functions by using (2.1) followed by formula (2.2) with $q=0$ we have that

$$
e_{1}[X] H_{\left(2^{x} 1^{y}\right)}[X ; t]=H_{\left(2^{x} 1^{y+1}\right)}[X ; t]+\left(1-t^{y}\right) H_{\left(2^{x+1} 1^{y-1}\right)}[X ; t]+\left(1-t^{x}\right) H_{\left(32^{x-1} 1^{y}\right)}[X ; t]
$$

Therefore, arriving at a formula for $H_{\left(32^{a} 1^{b}\right)}[X ; q, t]$ is a matter of applying formulas (2.1) and (2.10) to (2.6). Unfortunately, the result is not nearly as nice as it was in the case of (2.1).

\section{Proposition 2.1}

$$
\begin{aligned}
H_{\left(32^{a} 1^{b}\right)}[X ; q, t]= & \sum_{i=0}^{a} c_{i}^{(a, b)}\left(1-q^{2} t^{a+b+1}\right)\left(1-q t^{a+1}\right) H_{\left(32^{i} 1^{2 a+b-2 i}\right)}[X ; t]+q^{a+3} H_{\left(1^{2 a+b+3}\right)}[X ; t] \\
& +\sum_{i=1}^{a+2} c_{i}^{(a, b)} q\left(\frac{\left(1-q^{2} t^{a+b+1}\right)\left(1-q t^{a+1}\right)}{\left(1-t^{a+1-i}\right)\left(1-q t^{a+b+1-i}\right)}\right. \\
& +q \frac{\left(1-q^{2} t^{a+b+1}\right)\left(1-q t^{a+1}\right)\left(1-t^{i}\right)\left(1-t^{2 a+b+4-2 i}\right)}{\left(1-q t^{a+1+b-i}\right)\left(1-t^{a+1-i}\right)\left(1-t^{a+2-i}\right)\left(1-q t^{a+2+b-i}\right)} \\
& -q \frac{\left(1-q^{2} t^{a+b+1}\right)\left(1-t^{b}\right)(1-q)\left(1-t^{a+2}\right)}{\left(1-t^{a+2-i}\right)\left(1-q t^{a+1+b-i}\right)\left(1-t^{a+1-i}\right)\left(1-q t^{b}\right)} \\
& \left.-\frac{\left(1-q^{2} t^{b}\right)\left(1-q t^{a+1}\right)(1-q)\left(1-q t^{a+b+2}\right)}{\left(1-q t^{a+b+2-i}\right)\left(1-q t^{a+1+b-i}\right)\left(1-t^{a+1-i}\right)\left(1-q t^{b}\right)}\right) \\
& H_{\left(2^{i} 1^{2 a+b+3-2 i}\right)}[X ; t]
\end{aligned}
$$


A slightly simpler expression for this result can be given after the vertex operator is introduced, but it is not obvious when the coefficients of Proposition 2.1 are in a reduced form.

The proof of Proposition 2.1 uses the following lemmas that each follows from a simple calculation from the definition of $c_{i}^{(a, b)}=q^{a-i}\left(q t^{a+b-i+1} ; t\right)_{i} \frac{(t ; t)_{a}}{(t ; t)_{i}(t ; t)_{a-i}}$.

Lemma 2.2 For $0 \leq z<x$

$$
\begin{aligned}
& c_{z}^{(x, y)}=c_{z}^{(x, y-1)} \frac{\left(1-q t^{x+y}\right)}{\left(1-q t^{x+y-z}\right)} \\
& c_{z}^{(x, y)}=c_{z+1}^{(x, y)} q \frac{\left(1-t^{z+1}\right)}{\left(1-t^{x-z}\right)\left(1-q t^{x+y-z}\right)} \\
& c_{z}^{(x, y)}=c_{z}^{(x-1, y)} q \frac{\left(1-q t^{x+y}\right)\left(1-t^{x}\right)}{\left(1-q t^{x+y-z}\right)\left(1-t^{x-z}\right)}
\end{aligned}
$$

There are no tricks involved in the reduction of (2.6) to Proposition 2.1, just algebraic manipulation. Hence we leave the details of the proof to the reader who may be able to discover a better expression. This proposition is necessary only for comparison to a similar expression for $H_{3}^{q t} H_{\left(2^{a} 1^{b}\right)}[X ; q, t]$. o

To derive a formula for $H_{\left(42^{a} 1^{b}\right)}[X ; q, t]$ we will use the same brute force method for finding equations for the coefficients of the Hall-Littlewood symmetric functions. If we add a horizontal strip of size 2 on a two column Macdonald summetric function by multiplying by $g_{2}[X ; q, 0]$ where $g_{r}[X ; q, t]$ is defined in [19, eq. 2.8, p. 311] then we have the following terms

$$
\begin{aligned}
g_{2}[X ; q, 0] H_{\left(2^{a+1} 1^{b}\right)}[X ; q, t]= & A H_{\left(2^{a+2} 1^{b}\right)}[X ; q, t]+B H_{\left(32^{a+1} 1^{b-1}\right)}[X ; q, t]+ \\
& C H_{\left(32^{a} 1^{b+1}\right)}[X ; q, t]+D H_{\left(42^{a} 1^{b}\right)}[X ; q, t]
\end{aligned}
$$

where $A, B, C$, and $D$ are given as 


$$
\begin{aligned}
& A=\frac{1}{\left(1-q^{2} t^{a+b+1}\right)\left(1-q t^{a+1}\right)} \\
& B=\frac{\left(1-t^{b}\right)\left(1-q t^{a+b+1}\right)}{(1-q)\left(1-q^{2} t^{a+1}\right)\left(1-q t^{b}\right)\left(1-q^{2} t^{a+b+1}\right)} \\
& C=\frac{\left(1-t^{a+1}\right)\left(1-q^{2} t^{b}\right)}{(1-q)\left(1-q t^{b}\right)\left(1-q^{3} t^{a+b+1}\right)\left(1-q t^{a+1}\right)} \\
& D=\frac{\left(1-t^{a+1}\right)\left(1-q t^{a+b+1}\right)}{(1-q)\left(1-q^{2}\right)\left(1-q^{3} t^{a+b+1}\right)\left(1-q^{2} t^{a+1}\right)}
\end{aligned}
$$

Rearranging terms, we have that

$$
\begin{aligned}
H_{\left(42^{a} 1^{b}\right)}[X ; q, t]= & A^{\prime} H_{\left(2^{a+2} 1^{b}\right)}[X ; q, t]+B^{\prime} H_{\left(32^{a+1} 1^{b-1}\right)}[X ; q, t]+ \\
& C^{\prime} H_{\left(32^{a} 1^{b+1}\right)}[X ; q, t]+D^{\prime} g_{2}[X ; q, 0] H_{\left(2^{a+1} 1^{b}\right)}[X ; q, t]
\end{aligned}
$$

where $A^{\prime}, B^{\prime}, C^{\prime}$, and $D^{\prime}$ are given as

$$
\begin{aligned}
A^{\prime} & =-\frac{(1-q)\left(1-q^{2}\right)\left(1-q^{3} t^{a+b+1}\right)\left(1-q^{2} t^{a+1}\right)}{\left(1-q^{2} t^{a+b+1}\right)\left(1-q t^{a+1}\right)\left(1-t^{a+1}\right)\left(1-q t^{a+b+1}\right)} \\
B^{\prime} & =-\frac{\left(1-t^{b}\right)\left(1-q^{2}\right)\left(1-q^{3} t^{a+b+1}\right)}{\left(1-q t^{b}\right)\left(1-q^{2} t^{a+b+1}\right)\left(1-t^{a+1}\right)} \\
C^{\prime} & =-\frac{\left(1-q^{2} t^{b}\right)\left(1-q^{2}\right)\left(1-q^{2} t^{a+1}\right)}{\left(1-q t^{b}\right)\left(1-q t^{a+1}\right)\left(1-q t^{a+b+1}\right)} \\
D^{\prime} & =\frac{(1-q)\left(1-q^{2}\right)\left(1-q^{3} t^{a+b+1}\right)\left(1-q^{2} t^{a+1}\right)}{\left(1-t^{a+1}\right)\left(1-q t^{a+b+1}\right)}
\end{aligned}
$$

Only the last term in this expression does not have an expression in terms of HallLittlewood symmetric functions yet. As in the $\left(32^{a} 1^{b}\right)$ case, this can be computed by translating [19, p. 340, eq. 6.24(i)] and setting $q=0$. We have that

$$
\begin{aligned}
h_{2}[X] H_{\left(2^{x} 1^{y}\right)}[X ; t]= & H_{\left(2^{x+1} 1^{y}\right)}+\left(1-t^{y}\right) H_{\left(32^{x} 1^{y-1}\right)}[X ; t]+ \\
& \left(1-t^{x}\right) H_{\left(32^{x-1} 1^{y+1}\right)}[X ; t]+\left(1-t^{x}\right) H_{\left(42^{x-1} 1^{y}\right)}[X ; t]
\end{aligned}
$$


and

$$
\begin{aligned}
h_{1}[X]^{2} H_{\left(2^{x} 1^{y}\right)}[X ; t]= & H_{\left(2^{x} 1^{y+2}\right)}[X ; t] \\
& +\left(2-t^{y+1}-t^{y}\right) H_{\left(2^{x+1} 1^{y}\right)}[X ; t] \\
& +\left(1-t^{y}\right)\left(1-t^{y-1}\right) H_{\left(2^{x+2} 1^{y-2}\right)}[X ; t] \\
& +2\left(1-t^{x}\right) H_{\left(32^{x-1} 1^{y+1}\right)}[X ; t] \\
& +\left(1-t^{y}\right)\left(2-t^{x+1}-t^{x}\right) H_{\left(32^{x} 1^{y-1}\right)}[X ; t] \\
& +\left(1-t^{x}\right)\left(1-t^{x-1}\right) H_{\left(332^{x-2} 1^{y}\right)}[X ; t] \\
& +\left(1-t^{x}\right)(1-t) H_{\left(42^{x-1} 1^{y}\right)}[X ; t]
\end{aligned}
$$

and

$$
g_{2}[X ; q, 0]=\frac{q}{(1-q)\left(1-q^{2}\right)} h_{1}^{2}[X]+\frac{1}{1-q^{2}} h_{2}[X]
$$

The next proposition follows by taking coefficients in the formulas above.

Proposition 2.3 Let $E^{\prime}=\frac{D^{\prime}}{(1-q)\left(1-q^{2}\right)}$ and denote the coefficient of $H_{\left(32^{i} 1^{2 a+b-2 i}\right)}[X ; t]$ in $H_{\left(32^{a} 1^{b}\right)}[X ; q, t]$ (as given in Proposition [2.1) by $d_{i}^{(a, b)}$ and the coefficient of $H_{\left(2^{i} 1^{3+2 a+b-2 i}\right)}[X ; t]$ in $H_{\left(32^{a} 1^{b}\right)}[X ; q, t]$ by $e_{i}^{(a, b)}$. The coefficients for the symmetric function $H_{\mu}[X ; t]$ in the Macdonald symmetric function $H_{\left(42^{a} 1^{b}\right)}[X ; q, t]$ are given by the following table of expressions.

$$
\begin{array}{cl}
\mu=\left(2^{i} 1^{4+2 a+b-2 i}\right) & A^{\prime} c_{i}^{(a+2, b)}+B^{\prime} e_{i}^{(a+1, b-1)}+C^{\prime} e_{i}^{(a, b+1)}+E^{\prime}(1-q) c_{i-1}^{(a+1, b)}+ \\
& q E^{\prime}\left(c_{i}^{(a+1, b)}+\left(2-t^{2 a+b-2 i+5}-t^{2 a+b-2 i+4}\right) c_{i-1}^{(a+1, b)}\right. \\
& \left.\quad+\left(1-t^{2 a+b-2 i+6}\right)\left(1-t^{2 a+b-2 i+5}\right) c_{i-2}^{(a+1, b)}\right) \\
\mu=\left(32^{i} 1^{1+2 a+b-2 i}\right) & B^{\prime} d_{i}^{(a+1, b-1)}+C^{\prime} d_{i}^{(a, b+1)}+ \\
& E^{\prime}(1-q)\left(\left(1-t^{2 a+b+2-2 i}\right) c_{i}^{(a+1, b)}+\left(1-t^{i+1}\right) c_{i+1}^{(a+1, b)}\right)+ \\
& q E^{\prime}\left(2\left(1-t^{i+1}\right) c_{i+1}^{(a+1, b)}+\left(1-t^{2 a+b+2-2 i}\right)\left(2-t^{i+1}-t^{i}\right) c_{i}^{(a+1, b)}\right) \\
\mu=\left(332^{i} 1^{2 a+b-2 i-2}\right) & q E^{\prime}\left(1-t^{i+2}\right)\left(1-t^{i+1}\right) c_{i+2}^{(a+1, b)} \\
& E^{\prime}\left(1-t^{i+1}\right)(1-q t) c_{i+1}^{(a+1, b)}
\end{array}
$$

where if $j<0$ then $c_{j}^{(a, b)}=0$ and the coefficients $c_{i}^{(a, b)}, A^{\prime}, B^{\prime}, C^{\prime}$ and $D^{\prime}$ are all given in the text above. For all $\mu$ that do not follow a pattern in this table, the coefficient of $H_{\mu}[X ; t]$ in $H_{\left(42^{a} 1^{b}\right)}[X ; q, t]$ is zero. 
Although using this technique for computing expansions of Macdonald polynomials might be used with other bases, here it seems that a general formula for the expansion of $H_{\mu}[X ; q, t]$ in terms of $H_{\mu}[X ; t]$ will not be useful since patterns in the coefficients do not seem to exist as they did in the case when $\mu=\left(2^{a} 1^{b}\right)$. We will show that these two particular expansions do have a use to make the computation of Macdonald polynomials in these special cases much easier.

\section{The Vertex operator $H_{3}^{q t}$}

Our purpose in giving an expression for $H_{\left(32^{a} 1^{b}\right)}[X ; q, t]$ and $H_{\left(42^{a} 1^{b}\right)}[X ; q, t]$, is to show that the expressions given in Theorems 1.3 and 1.4 have the properties $H_{3}^{q t} H_{\left(2^{a} 1^{b}\right)}[X ; q, t]=$ $H_{\left(32^{a} 1^{b}\right)}[X ; q, t]$ and $H_{4}^{q t} H_{\left(2^{a} 1^{b}\right)}[X ; q, t]=H_{\left(42^{a} 1^{b}\right)}[X ; q, t]$.

The vertex operators $H_{3}^{q t}$ and $H_{4}^{q t}$ are given as expressions using the $H_{m}^{t}$ and $\bar{H}_{m}^{t}$ operators and so we will need the action of these operators on the Hall-Littlewood basis.

\section{Lemma 3.1}

$$
\begin{aligned}
\bar{H}_{3}^{t} H_{\left(2^{x} 1^{y}\right)}[X ; t]= & t^{x} H_{\left(2^{x} 1^{y+3}\right)}[X ; t]-t^{x+y+1}(1+t) H_{\left(2^{x+1} 1^{y+1}\right)}[X ; t] \\
& -t^{x+y+1}\left(1-t^{y}\right) H_{\left(2^{x+2} 1^{y-1}\right)}[X ; t]+t^{2 x+y+1} H_{\left(32^{x} 1^{y}\right)}[X ; t]
\end{aligned}
$$

Proof: We note the following three commutation relations:

$$
\begin{aligned}
\bar{H}_{n}^{t} H_{m}^{t} & =t^{m-1} H_{m}^{t} \bar{H}_{n}^{t} \\
H_{m-1}^{t} H_{n}^{t} & =t H_{m}^{t} H_{n-1}^{t}+t H_{n}^{t} H_{m-1}^{t}-H_{n-1}^{t} H_{m}^{t} \\
H_{m}^{t} H_{m+1}^{t} & =t H_{m+1}^{t} H_{m}^{t}
\end{aligned}
$$

A proof of (3.2) is in [19, p. 238] and (3.3) is a specialization of that. The proof of equation (3.1) is the same as [22, Lemma 2.4].

For (3.2), in particular we have that

$$
H_{1}^{t} H_{3}^{t}=t H_{3}^{t} H_{1}^{t}-(1-t) H_{2}^{t} H_{2}^{t}
$$

And $\bar{H}_{3} H_{\left(2^{x} 1^{y}\right)}[X ; t]$ can be computed by calculating commutation relations.

$$
\begin{aligned}
\bar{H}_{3} H_{\left(2^{x} 1^{y}\right)}[X ; t]= & t^{x}\left(H_{2}^{t}\right)^{x} \bar{H}_{3}^{t} H_{\left(1^{y}\right)}[X ; t] \\
= & t^{x}\left(H_{2}^{t}\right)^{x}\left(H_{1}^{t}\right)^{y} \bar{H}_{3}^{t} 1 \\
= & t^{x}\left(H_{2}^{t}\right)^{x}\left(H_{1}^{t}\right)^{y}\left(H_{\left(1^{3}\right)}[X ; t]-\left(t+t^{2}\right) H_{(21)}[X ; t]+t^{2} H_{(3)}[X ; t]\right) \\
= & t^{x} H_{\left(2^{x} 1^{y+3}\right)}[X ; t]-t^{x}\left(t+t^{2}\right) t^{y+1} H_{\left(2^{x+1} 1^{y+1}\right)}[X ; t] \\
& -t^{x+2} t^{y-1}\left(1-t^{y}\right) H_{\left(2^{x+2} 1^{y-1}\right)}[X ; t]+t^{x+2} t^{x+y-1} H_{\left(32^{x} 1^{y}\right)}[X ; t]
\end{aligned}
$$


We now give an outline of the proof of Theorem 1.3.

Proof: We use equation (2.1) and Lemma 3.1 and show that the expression is equivalent to Proposition 2.1.

$$
\begin{aligned}
H_{3}^{q t} H_{\left(2^{a} 1^{b}\right)}[X ; q, t]= & (1-q) H_{3}^{t} H_{\left(2^{a} 1^{b}\right)}[X ; q, t]+q e_{1}[X] H_{\left(2^{a+1} 1^{b}\right)}[X ; q, t] \\
& +q^{2}(q-1) \bar{H}_{3}^{t} H_{\left(2^{a} 1^{b}\right)}[X ; q, t] \\
= & (1-q) \sum_{i=0}^{a} c_{i}^{(a, b)} H_{\left(32^{i} 1^{2 a+b-2 i}\right)}[X ; t] \\
+ & q \sum_{i=0}^{a+1} c_{i}^{(a+1, b)} e_{1}[X] H_{\left(2^{i} 1^{2 a+2+b-2 i}\right)}[X ; t] \\
+ & q^{2}(q-1) \sum_{i=0}^{a} c_{i}^{(a, b)}\left(t^{i} H_{\left(2^{i} 1^{2 a+b-2 i+3}\right)}[X ; t]\right. \\
& -t^{2 a+b-i+1}(1+t) H_{\left(2^{i+1} 1^{2 a+b-2 i+1}\right)}[X ; t] \\
& -t^{2 a+b-i+1}\left(1-t^{2 a+b-2 i}\right) H_{\left(2^{i+2} 1^{2 a+b-2 i-1}\right)}[X ; t] \\
& \left.\left.+t^{2 a+b+2} H_{\left(32^{i} 1^{2 a+b-2 i}\right)}[X ; t]\right)\right)
\end{aligned}
$$

From here it is only algebraic manipulation to reduce the expression to one like

$$
\begin{aligned}
& =\sum_{i=0}^{a} c_{i}^{(a, b)}\left(1-q^{2} t^{a+b+1}\right)\left(1-q t^{a+1}\right) H_{\left(32^{i} 1^{2 a+b-2 i}\right)}[X ; t]+q^{a+3} H_{\left(1^{2 a+b+3}\right)}[X ; t] \\
& +\sum_{i=1}^{a+2}\left(q c_{i}^{(a+1, b)}+\left(1-t^{2 a+b+4-2 i}\right) q c_{i-1}^{(a+1, b)}\right. \\
& \quad+q^{2}(q-1) c_{i}^{(a, b)} t^{i}-q^{2}(q-1) c_{i-1}^{(a, b)} t^{2 a+b+2-i}(1+t) \\
& \left.\quad-q^{2}(q-1) c_{i-2}^{(a, b)} t^{2 a+b+3-i}\left(1-t^{2 a+b+4-2 i}\right)\right) H_{\left(2^{i} 1^{2 a+b+3-2 i}\right)}[X ; t]
\end{aligned}
$$

by converting all coefficients in terms of $c_{i}^{(a, b)}$ using equations (2.12), (2.13) and (2.14) we derive the following expression 


$$
\begin{aligned}
& =\sum_{i=0}^{a} c_{i}^{(a, b)}\left(1-q^{2} t^{a+b+1}\right)\left(1-q t^{a+1}\right) H_{\left(32^{i} 1^{2 a+b-2 i}\right)}[X ; t]+q^{a+3} H_{\left(1^{2 a+b+3}\right)}[X ; t] \\
& +\sum_{i=1}^{a+2} q c_{i}^{(a, b)}\left(q \frac{\left(1-q t^{a+1+b}\right)\left(1-t^{a+1}\right)}{\left(1-q t^{a+1+b-i}\right)\left(1-t^{a+1-i}\right)}\right. \\
& \quad+q^{2} \frac{\left(1-t^{2 a+b+4-2 i}\right)\left(1-q t^{a+1+b}\right)\left(1-t^{a+1}\right)\left(1-t^{i}\right)}{\left(1-q t^{a+1+b-i}\right)\left(1-t^{a+1-i}\right)\left(1-t^{a+2-i}\right)\left(1-q t^{a+2+b-i}\right)} \\
& \quad+q(q-1) t^{i}-q^{2}(q-1) \frac{\left(1-t^{i}\right)}{\left(1-q t^{a+b+1-i}\right)\left(1-t^{a+1-i}\right)} t^{2 a+b+2-i}(1+t) \\
& \left.\quad-q^{3}(q-1) \frac{\left(1-t^{i}\right)\left(1-t^{i-1}\right) t^{2 a+b+3-i}\left(1-t^{2 a+b+4-2 i}\right)}{\left(1-q t^{a+b+2-i}\right)\left(1-t^{a+2-i}\right)\left(1-q t^{a+b+1-i}\right)\left(1-t^{a+1-i}\right)}\right) H_{\left(2^{i} 1^{2 a+b+3-2 i}\right)}[X ; t]
\end{aligned}
$$

which can be shown to be equivalent to Proposition 2.1 by more algebraic manipulation or by appealing to a computer algebra package such as Mathematica. $\circ$

As a corollary, (3.4) is an expression for the expansion of $H_{\left(32^{a} 1^{b}\right)}[X ; q, t]$ in terms of the basis $H_{\mu}[X ; t]$ since it seems to be a slightly nicer expression for the Macdonald polynomials than either (2.11) or (3.5).

We make the following substitution of notation to indicate what the pieces of this operator represent. Define the following operators

$$
\begin{aligned}
& H^{\text {[2]13 }}=H_{3}^{t} \quad H^{[\text {[1] }}=e_{1} H_{2}^{t}-H_{3}^{t} \\
& H^{\text {通 }}=e_{1} \bar{H}_{2}^{t}-\bar{H}_{3}^{t} \quad H^{\frac{\sqrt[3]{2}}{4}}=\bar{H}_{3}^{t}
\end{aligned}
$$

Then notice that $H_{3}^{q t}$ has the expression

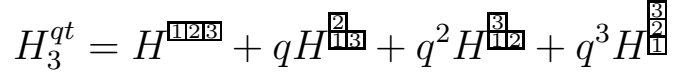

Suddenly the operator that has so far been very unelegant, looks like it is shaping up. In the following sections we will show that not only is $H_{3}^{q t} H_{\left(2^{a} 1^{b}\right)}[X ; q, t]$ Schur positive, but so are all of the pieces of this expression $\left(H^{[1213} H_{\left(2^{a} 1^{b}\right)}[X ; q, t], H^{[1] 3} H_{\left(2^{a} 1^{b}\right)}[X ; q, t]\right.$, $H^{\text {通 }} H_{\left(2^{a} 1^{b}\right)}[X ; q, t]$, and $\left.H^{\frac{3}{2}} H_{\left(2^{a} 1^{b}\right)}[X ; q, t]\right)$ and that each is a generating function for a subclass of standard tableaux. 


\section{The Vertex operator $H_{4}^{q t}$}

As with the vertex operator $H_{3}^{q t}$, we will use what we know about the action of $H_{m}^{t}$ and $\bar{H}_{m}^{t}$ on the Hall-Littlewood basis to compute the action of $(1-q)\left(1-q^{2}\right)\left(H_{4}^{t}+q^{3} \bar{H}_{4}^{t}\right)+q(1+$ q) $e_{1} H_{3}^{q t}-q^{2}\left(e_{2}+q h_{2}\right) H_{2}^{q t}$ on equation (2.1). The only piece of this equation that we have not already given an expression for is the action of $\bar{H}_{4}^{t}$ on the Hall-Littlewood symmetric functions.

Lemma 4.1 For $a, b \geq 0$ we have that

$$
\begin{aligned}
\bar{H}_{4}^{t} H_{\left(2^{a} 1^{b}\right)}[X ; t]= & t^{a} H_{\left(2^{a} 1^{b+4}\right)}[X ; t]-t^{a+b+1}\left(1+t+t^{2}\right) H_{\left(2^{a+1} 1^{b+2}\right)}[X ; t] \\
& -t^{a+b+1}\left(1+t-t^{b}-t^{b+1}-t^{b+2}\right) H_{\left(2^{a+2} 1^{b}\right)}[X ; t] \\
& -t^{a+b+1}\left(1-t^{b-1}\right)\left(1-t^{b}\right) H_{\left(2^{a+3} 1^{b-2}\right)}[X ; t] \\
& +t^{2 a+b+2}(1+t) H_{\left(32^{a} 1^{b+1}\right)}[X ; t]+t^{2 a+b+2}(1+t)\left(1-t^{b}\right) H_{\left(32^{a+1} 1^{b-1}\right)}[X ; t] \\
& +t^{2 a+b+2}\left(1-t^{a}\right) H_{\left(332^{a-1} 1^{b}\right.}[X ; t]-t^{2 a+b+3} H_{\left(42^{a} 1^{b}\right)}
\end{aligned}
$$

Proof: First set $a=0$, and prove that for all $b$ greater than or equal to zero we have that the theorem is true by induction using the identities (3.1), (3.3), and (3.2) in the special cases of $\bar{H}_{4}^{t} H_{1}^{t}=t H_{1}^{t} \bar{H}_{4}^{t}, H_{1}^{t} H_{4}^{t}=t H_{4}^{t} H_{1}^{t}+\left(t^{2}-1\right) H_{3}^{t} H_{2}^{t}, H_{1}^{t} H_{3}^{t}=t H_{3}^{t} H_{1}^{t}+(t-1) H_{2}^{t} H_{2}^{t}$, and $H_{1}^{t} H_{2}^{t}=t H_{2}^{t} H_{1}^{t}$. The base case is a calculation of

$\bar{H}_{4}^{t}(1)=H_{\left(1^{4}\right)}[X ; t]-t\left(1+t+t^{2}\right) H_{(211)}[X ; t]+t^{3} H_{(22)}[X ; t]+t^{2}(1+t) H_{(31)}[X ; t]-t^{3} H_{(4)}[X ; t]$

Now for $a>0$ we have that

$$
\bar{H}_{4}^{t} H_{\left(2^{a} 1^{b}\right)}[X ; t]=t H_{2}^{t} \bar{H}_{4}^{t} H_{\left(2^{a-1} 1^{b}\right)}[X ; t]
$$

Using the relations $H_{2}^{t} H_{3}^{t}=t H_{3}^{t} H_{2}^{t}$ and $H_{2}^{t} H_{4}^{t}=H_{4}^{t} H_{2}^{t}+(t-1) H_{3}^{t} H_{3}^{t}$, the result follows. $\circ$

We are now ready to prove the vertex operator property.

Proof: $\quad$ (of Theorem 1.4)

For each $\mu$ that appears in Proposition 2.3 we take the coefficient of $H_{\mu}[X ; t]$ in the expression

$$
\begin{aligned}
H_{4}^{q t} H_{\left(2^{a} 1^{b}\right)}[X ; q, t]= & (1-q)\left(1-q^{2}\right)\left(H_{4}^{t}+q^{3} \bar{H}_{4}^{t}\right) H_{\left(2^{a} 1^{b}\right)}[X ; q, t]+q(1+q) e_{1}[X] H_{\left(32^{a} 1^{b}\right)}[X ; q, t] \\
& -q^{2}\left(h_{1}^{2}[X]+(q-1) h_{2}[X]\right) H_{\left(2^{a+1} 1^{b}\right)}[X ; q, t]
\end{aligned}
$$


We need Proposition 2.1] equations (2.1), (2.10), (2.25), (2.26) and (4.1), and one more translation of the Macdonald-Pieri rule.

$$
\begin{aligned}
e_{1}[X] H_{\left(32^{a} 1^{b}\right)}[X ; t]= & H_{\left(32^{a} 1^{b+1}\right)}[X ; t]+\left(1-t^{b}\right) H_{\left(32^{a+1} 1^{b-1}\right)}[X ; t] \\
& +\left(1-t^{a}\right) H_{\left(332^{a-1} 1^{b}\right)}[X ; t]+(1-t) H_{\left(42^{a} 1^{b}\right)}[X ; t]
\end{aligned}
$$

As in Proposition 2.3, denote the coefficient of $H_{\left(32^{i} 1^{2 a+b-2 i}\right)}[X ; t]$ in $H_{\left(32^{a} 1^{b}\right)}[X ; q, t]$ (as given in Proposition 2.1) by $d_{i}^{(a, b)}$ and the coefficient of $H_{\left(2^{i} 1^{3+2 a+b-2 i}\right)}[X ; t]$ in $H_{\left(32^{a} 1^{b}\right)}[X ; q, t]$ by $e_{i}^{(a, b)}$.

If $\mu=\left(42^{i} 1^{2 a+b-2 i}\right)$, the coefficient is

$$
(1-q)\left(1-q^{2}\right)\left(1-t^{2 a+b+3} q^{3}\right) c_{i}^{(a, b)}+q(1+q)(1-t) d_{i}^{(a, b)}+q^{2}\left(1-t^{i+1}\right)(t-q) c_{i+i}^{(a+1, b)}
$$

If $\mu=\left(332^{i} 1^{2 a+b-2-2 i}\right)$, then the coefficient is

$$
(1-q)\left(1-q^{2}\right) q^{3} t^{2 a+b+2}\left(1-t^{i+1}\right) c_{i+1}^{(a, b)}+q(1+q)\left(1-t^{i+1}\right) d_{i+1}^{(a, b)}-q^{2}\left(1-t^{i+2}\right)\left(1-t^{i+1}\right) c_{i+2}^{(a+1, b)}
$$

If $\mu=\left(32^{i} 1^{2 a+b+1-2 i}\right)$, then the coefficient is

$$
\begin{aligned}
& (1-q)\left(1-q^{2}\right) q^{3} t^{2 a+b+2}(1+t)\left(c_{i}^{(a, b)}+\left(1-t^{2 a+b+2-2 i}\right) c_{i-1}^{(a, b)}\right) \\
& +q(1+q)\left(\left(1-t^{i+1}\right) e_{i+1}^{(a, b)}+d_{i}^{(a, b)}+\left(1-t^{2 a+b+2-2 i}\right) d_{i-1}^{(a, b)}\right) \\
& -q^{2}\left(2\left(1-t^{i+1}\right) c_{i+1}^{(a+1, b)}+\left(1-t^{2 a+b+2-2 i}\right)\left(2-t^{i+1}-t^{i}\right) c_{i}^{(a+1, b)}\right. \\
& \left.+(q-1)\left(\left(1-t^{2 a+b+2-2 i}\right) c_{i}^{(a+1, b)}+\left(1-t^{i+1}\right) c_{i+1}^{(a+1, b)}\right)\right)
\end{aligned}
$$

Finally, if $\mu=\left(2^{i} 1^{2 a+b+4-2 i}\right)$, then the coefficient is

$$
\begin{aligned}
& (1-q)\left(1-q^{2}\right) q^{3}\left(t^{i} c_{i}^{(a, b)}-t^{2 a+b+2-i}\left(1+t+t^{2}\right) c_{i-1}^{(a, b)}\right. \\
& -t^{2 a+b+3-i}\left(1+t-t^{2 a+b+4-2 i}-t^{2 a+b+5-2 i}-t^{2 a+b+6-2 i}\right) c_{i-2}^{(a, b)} \\
& \left.-t^{2 a+b+4-i}\left(1-t^{2 a+b+5-2 i}\right)\left(1-t^{2 a+b+6-2 i}\right) c_{i-3}^{(a, b)}\right) \\
& +q(1+q)\left(e_{i}^{(a, b)}+\left(1-t^{2 a+b+5-2 i}\right) e_{i-1}^{(a, b)}\right) \\
& -q^{2}\left(c_{i}^{(a+1, b)}+\left(2-t^{2 a+b+5-2 i}-t^{2 a+b+4-2 i}\right) c_{i-1}^{(a+1, b)}\right. \\
& +\left(1-t^{2 a+b+6-2 i}\right)\left(1-t^{2 a+b+5-2 i}\right) c_{i-2}^{(a+1, b)} \\
& \left.+(q-1) c_{i-1}^{(a+1, b)}\right)
\end{aligned}
$$


These can be shown to be equivalent to Proposition 2.3 by hand (with an enormous amount of patience) or by reducing these expressions on computer using a computer algebra package such as Mathematica. $\circ$

We introduce the following notation as we did in the case of $H_{3}^{q t}$ to better demonstrate the structure of this operator. Define the operators

$$
\begin{aligned}
& H^{\text {[12131] }}=H_{4}^{t} \\
& H \text { 橉 }+H^{\text {盘田 }}=h_{2} H_{2}^{t}-H_{4}^{t} \\
& H^{\text {界细 }}=e_{1} H_{3}^{t}-H_{4}^{t} \\
& H \text { 睡 }=e_{2} H_{2}^{t}-e_{1} H_{3}^{t}+H_{4}^{t} \\
& H_{\text {团213 }}=h_{2} \bar{H}_{2}^{t}-h_{1} \bar{H}_{3}^{t}+\bar{H}_{4}^{t} \\
& H \text { 周 }=e_{1} \bar{H}_{3}^{t}-\bar{H}_{4}^{t} \quad H \text { 章 }=\bar{H}_{4}^{t}
\end{aligned}
$$

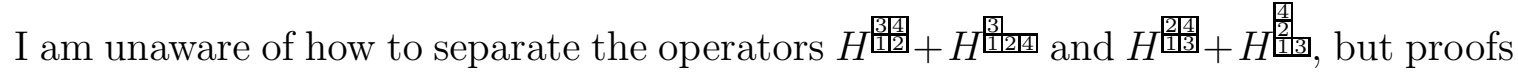
will work nearly as expected as long as we consider these as two single entities. Notice that $H_{4}^{q t}$ has the expression

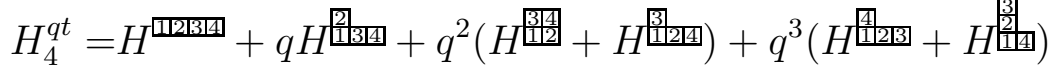

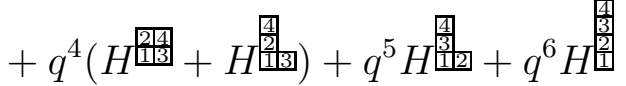

These operators (and the ones for $H_{3}^{q t}$ ) were all defined so that they have the relation $\left.\omega H^{T} \omega\right|_{t \rightarrow 1 / t} R^{t}=H^{\omega T}$ (where $\omega T$ represents the diagram flipped about its diagonal).

Using the relation $H_{\mu^{\prime}}[X ; q, t]=\omega H_{\mu}[X ; t, q]$, we note that $\omega H_{2}^{t q} \omega, \omega H_{3}^{t q} \omega$ and $\omega H_{4}^{t q} \omega$ add a column of size 2, 3 and 4 respectively to a Macdonald polynomial indexed by a two row partition.

These vertex operator formulas provide a fast method of computation on a computer algebra package (such as Maple) of the Macdonald polynomials for partitions with two rows, two columns, or of the form $\left(32^{a} 1^{b}\right),(a b 1),\left(42^{a} 1^{b}\right)$ or $\left(a b 1^{2}\right)$. Theorem 1.2, 1.3 and 1.4 are therefore enough to calculate all of the Macdonald polynomials and the $(q, t)$-Kostka coefficients through $n=7$ and all but one partition at $n=8,(3,3,2)$.

The operators $H_{2}^{q t}, H_{3}^{q t}$ and $H_{4}^{q t}$ can be modified so that they work on the other Macdonald bases. Let $V$ be the operator with the property $V J_{\mu}[X ; q, t]=H_{\mu}[X ; q, t]$. 
Define $J_{m}^{q t}=V^{-1} H_{m}^{q t} V$ then $J_{m}^{q t}$ has the corresponding property on the $J_{\mu}[X ; q, t]$ basis. The formulas seem to take on an interesting form when expressed in this way.

The methods presented here might be used to handle a few additional cases of deriving formulas for vertex operators for Macdonald polynomials, but experimental computations indicate that using the Hall-Littlewood vertex operators will not give nice expressions in general. There seems to be another family of vertex operators that has the Hall-Littlewood vertex operators as a special case that might be used to show that the $(q, t)$-Kostka coefficients are polynomials with non-negative integer coefficients.

\section{Tableaux, charge, and two column Macdonald poly- nomials}

A column strict tableau is a diagram of a partition (or skew partition) with each cell labeled with a positive integer such that the labels increase weakly traveling from left to right in the rows and the labels increase strictly traveling from bottom to top in the columns. A standard tableau is a column strict tableau with the numbers 1 to $n$ where $n$ is the size of the partition.

Let $T$ be a column strict tableau. Denote the shape of the tableau by $\lambda(T)$, the total number of cells in the diagram by $|T|$, and the number of cells labeled with an $i$ by the symbol $T_{i}$. The content of the tableau will be the tuple $\mu(T)=\left(T_{1}, T_{2}, \ldots, T_{h}\right.$ ) (where $h$ is the highest label that appears in the tableau). $\mathrm{T}$ is said to be of partition content if the content vector $\mu(T)$ is a partition. The content of a word is defined similarly (the tuple consisting of (the number of 1's in the word, the number of 2's in the word, etc.))

We begin by defining an algorithm for calculating the statistic called charge on column strict tableaux of partition weight. This statistic was introduced by Lascoux and Schützenberger.

First, charge is defined for words of content weight $\mu=1^{n}$. An index is given to each letter in the word. The index 0 is assigned to 1 . If the letter $i$ has index $k$ then the index of the letter $i+1$ is $k$ if $i+1$ lies to the left of $i$ and the index is $k+1$ if $i+1$ lies to the right of $i$. The charge of the word is defined to be the sum of the indices.

If $w$ is a word with content of partition weight then it is first broken up into standard subwords by the following procedure. Place an $x$ under the first 1 in the word traveling from right to left. Next place an $x$ under the first 2 traveling to the left from there. Continue

placing an $x$ underneath each of the letters 1 through $l(\mu(w))$ traveling from right to left and 
beginning again at the right side of the word each time the left hand side is reached. The first standard subword consists of the letters that have $x$ s underneath them read from left to right. Erase these letters to form a new word $w^{\prime}$ and repeat the procedure forming the next standard subword with the labels 1 through $l\left(\mu\left(w^{\prime}\right)\right)$. Stop when all letters have been erased. The charge of the word is then defined to be the sum of the charges of the standard subwords.

The reading word of a tableau is the word formed by reading the entries in the cells in each of the rows from left to right, starting with the top row. Denote the reading word of $T$ by $\mathcal{R}(T)$. Lastly, the charge of a tableau $\mathrm{T}$ is defined to be the charge of the reading word of $T$. Denote the charge of a word $w$ (or tableau $T$ ) by $c(w)$ (respectively $c(T)$ ).

\section{Example 5.1}

\begin{tabular}{|c|c|c|c|c|}
\hline 7 & & & & \\
\hline 3 & 4 & 6 & & \\
\hline 2 & 2 & 3 & 5 & \\
\hline 1 & 1 & 1 & 2 & \begin{tabular}{l|l}
4 & 8
\end{tabular} \\
\hline
\end{tabular}

A tableau of shape $\lambda=(6,4,3,1)$ and content $\mu=(3,3,2,2,1,1,1,1)$. The reading word of this tableau is 73462235111248. The word has standard subwords 73625148, 4231 and 12. The first standard subword has charge 6, the second charge 2, the third charge 1. The charge of the tableau is 9 .

If $x, y$ and $z$ are letters in the words $u$ and $v$ and $w$ and $\tilde{w}$ are subwords then say that two words $u$ and $v$ are elementary Knuth equivalent if either $u=w x z y \tilde{w}$ and $v=w z x y \tilde{w}$ where $x \leq y<z$ or $u=w y z x \tilde{w}$ and $v=w y x z \tilde{w}$ where $x<y \leq z$.

Next say that two words $u$ and $v$ are Knuth equivalent and write $u \sim v$ if they are in the symmetric, transitive, reflexive closure of the elementary Knuth equivalence.

There are several important facts about words and the charge of words that we will use to develop statistics here. These are well known results and proofs can be found in [18], [1], 2, 4.

Fact 5.2 Every word is equivalent to the reading word of a unique tableau with partition shape.

Fact 5.3 If $u \sim v$ then $c(u)=c(v)$. 
Fact 5.4 If $w=w_{1} 1^{a} w_{2}$ where $w_{1}$ and $w_{2}$ are subwords of $w$ that do not contain 1 then $c(w)=c\left(\left(w_{2} w_{1}\right) \downarrow^{1}\right)+\left|w_{2}\right|$ where the notation of $\downarrow^{k}$ (dually, $\left.\uparrow_{k}\right)$ indicates that the letters of the word to the left of this symbol have their labels decreased (increased) by $k$.

Fact 5.5 If $w=x_{1} x_{2} \cdots x_{n}$ is a standard word, then $c(w)=\left(\begin{array}{c}n \\ 2\end{array}\right)-c\left(x_{n} x_{n-1} \cdots x_{1}\right)$.

Fact 5.6 If $w=x_{1} x_{2} \cdots x_{n}$ is a standard word and $w$ is Knuth equivalent to the reading word of a standard tableau $T$, then $x_{n} x_{n-1} \cdots x_{1}$ is Knuth equivalent to the reading word of the conjugate tableau $\omega T$.

The algorithm of Robinson-Schenstead will be used implicitly throughout the following sections. Knowledge of the expressions row/column insertion/evacuation and their relations to Knuth equivalence are assumed in some of the algorithms. Frequently in the text we will identify a tableau with its reading word

It is due to the development of this theory and the results of Lascoux and Schützenberger [1], [18, 1], 23] that the Hall-Littlewood polynomials $H_{\mu}[X ; t]=\sum_{T \in C S T^{\mu}} t^{c(T)} s_{\lambda(T)}[X]$ where $C S T^{\mu}$ is the collection of column strict tableaux of content $\mu$.

To describe the standard tableaux statisics for the case that the Macdonald polynomials were indexed by $\left(2^{a} 1^{b}\right)$ we defined two procedures for building the standard tableaux of size $n+2$ from those of size $n$. The statement of these definitions will be for arbitrary $m$ but for now set $m=2$.

Let $T$ be a standard tableaux of shape $\lambda \vdash n$ and let $\rho$ be a partition of $2 n+m$ such that $\rho / \lambda \in \mathcal{H}_{n+m}$. Consider the cells that are in $\lambda$ and not in $\rho^{r}$ and perform one column evacuation for each cell from right to left so that cells are evacuated in increasing order. Let the row of evacuated cells be $R$ and the remaining tableau be $\tilde{T}$. Raise the labels of the cells of $R$ and $\tilde{T}$ by $m$ and row insert the labels 1 through $m$ followed by all of $R$ in increasing order. The result will be the definition of the tableau $\mathbf{H}_{m}^{\rho} T$.

We may say that if $\mathbf{H}_{m}^{\rho} T$ and $\rho$ are given, $T$ can be recovered by evacuating $12 \cdots m R$ a row of size $m+\left|\lambda / \rho^{r}\right|$ from $\mathbf{H}_{m}^{\rho} T$ to leave $\tilde{T}$ of shape $\rho^{r}$. Denote this reverse operation by $\left(\mathbf{H}_{m}^{\rho}\right)^{-1}$.

Also define the operator that adds a column block of $m$ cells to be the transpose of the operation $\mathbf{H}_{m}^{\rho} T$. Again, let $T$ be a standard tableaux of shape $\lambda \vdash n$. Let $\rho \vdash n+m$ be a partition with $\rho / \lambda \in \mathcal{V}_{n+m}$. Consider the cells that are in $\lambda$ and are not in $\rho^{c}$ and perform one row evacuation for each cell from top to bottom so that the cells are evacuated in increasing order. Let the column of evacuated cells be $C$ and the remaining tableau be 
$\tilde{T}$. Raise the labels of the cells of $C$ and $\tilde{T}$ by $m$ and column insert the labels 1 , through $m$ and all of $C$ in increasing order. The resulting tableau will be the definition of $\overline{\mathbf{H}}_{m}^{\rho} T$. For the same reason as before, $T$ can be recovered if $\overline{\mathbf{H}}_{m}^{\rho} T$ and $\rho$ are given.

Example 5.7 Let $T=$\begin{tabular}{|l|l|l|l|}
\hline 2 & 4 & \\
\hline 1 & 3 & 5 & 6 \\
\hline
\end{tabular}

Let $\rho=(11,3)$ then in the procedure we have that $R=$\begin{tabular}{|l|l|l|l|l|l|l|l|l}
2 & 4 & 5
\end{tabular} and $\tilde{T}=$\begin{tabular}{lll|l}
1 & 3 & 6
\end{tabular} .

The labels are all raised by two and 1, 2 and $R$ are all inserted into $\tilde{T}$ to form the tableau

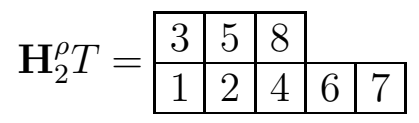

Consider $T$ is the same tableau, but now $\rho=\left(4,3,1^{7}\right)$. In the procedure for adding a column of size 2 we have that $C=6$ and $\tilde{T}=$\begin{tabular}{|l|l|l|}
2 & 4 & \\
\hline 1 & 3 & 5
\end{tabular} . Therefore,

$$
\overline{\mathbf{H}}_{2}^{\rho} T=\begin{array}{|l|l|l|l|}
\hline 8 & \multicolumn{3}{|l}{} \\
\hline 2 & 4 & 6 & \multicolumn{1}{|c}{} \\
\cline { 1 - 3 } 1 & 3 & 5 & 7 \\
\hline
\end{array}
$$

We also introduced the notion of the $\left(2^{a} 1^{b}\right)$ - type of a standard tableau. The type is defined recursively by 'unbuilding' the standard tableau in blocks of size 2 (for now set $m=2$ in the following definition).

If $112 \square \mathrm{m}$ is a subtableau of $T$ then let $R$ be the first row of $T$ and $\tilde{T}$ be $T$ with the first row of $T$ removed. Define $\mathbf{H}_{m}^{-1} T$ to be the tableau formed by column inserting the labels in $R$ that are not the lables 1 through $m$ into $\tilde{T}$ in decreasing order then lower all of the labels in the result by $m$.

If $\frac{m}{\frac{m}{2}}$ is a subtableau of $T$ then let $C$ be the first column of $T$ and $\tilde{T}$ be $T$ with the first column of $T$ removed. Define $\mathbf{H}_{m}^{-1} T$ to be the tableau formed by row inserting the labels in $C$ that are not a 1 through $m$ into $\tilde{T}$ in decreasing order then lower all of the labels in the result by $m$.

Now define the $\left(2^{a} 1^{b}\right)-$ type of $T$ as follows.

- If $a=0$ and $\mu=\left(1^{b}\right)$ then type $_{\mu}(T)=\left(\square^{b}\right)$.

- If $a>1$ and 2 lies to the right of 1 in $T$, then $\operatorname{type}_{\left(2^{a} 1^{b}\right)}(T)=\left(\right.$ 띠, type $\left.e_{\left(2^{a-1} 1^{b}\right)}\left(\mathbf{H}_{2}^{-1} T\right)\right)$

- If $a>1$ and 2 lies just above 1 in $T$, then $\operatorname{type}_{\left(2^{a} 1^{b}\right)}(T)=\left(\right.$ 원, type $e_{\left(2^{a-1} 1^{b}\right)}\left(\mathbf{H}_{2}^{-1} T\right)$ ) 
Example 5.8 Let $T=$\begin{tabular}{|l|l|l|}
\hline 3 & & \\
\hline 2 & 6 & \\
\hline 1 & 4 & 5 \\
\hline
\end{tabular} .

$\mathbf{H}_{2}^{-1} T=$\begin{tabular}{|l|l|l|}
\hline 4 & \\
\cline { 1 - 1 } 2 &
\end{tabular} and $\mathbf{H}_{2}^{-1} \mathbf{H}_{2}^{-1} T=$\begin{tabular}{lll}
1 & 2 \\
\hline 1 & 3 \\
\hline
\end{tabular}

Therefore, $\operatorname{type}_{\left(2^{3}\right)}(T)=($ 뉸, 뉸, 띠) .

It was necessary to introduce more machinery in [22] to prove the following results. Here we will take them as given without defining the handful of tableaux operators that were necessary to verify that they are true. The first is Proposition 3.5 and the second is Corollary 3.11 of 22$]$.

Proposition 5.9 Let $n=2 a+b$. The statistic $a_{\mu}(T)$ and $b_{\mu}(T)$ where $\mu=\left(2^{a} 1^{b}\right)$ from equation (1.5) may be defined to be

$$
\begin{aligned}
& a_{\mu}(T)=c(T)-\sum_{i=1}^{a}((n+1)-2 i) \chi\left(\text { type }_{\mu}(T)_{i}=\text { 띠 }\right) \\
& b_{\mu}(T)=\sum_{i=1}^{a} \chi\left(\operatorname{type}_{\mu}(T)_{i}=\text { 园 }\right)
\end{aligned}
$$

Proposition 5.10 Let $n=2 a+b$ and $T \in S T^{n}$ and let $\rho$ be a partition of $2 n+2$ such that $\rho / \lambda(T) \in \mathcal{H}_{n+2}$ then

$$
\operatorname{type}_{\left(2^{a+1} 1^{b}\right)}\left(\mathbf{H}_{2}^{\rho} T\right)=\left(\text { 띠, } \operatorname{type}_{\left(2^{a} 1^{b}\right)}(T)\right)
$$

Similarly, if $\rho / \lambda(T) \in \mathcal{V}_{n+2}$ then

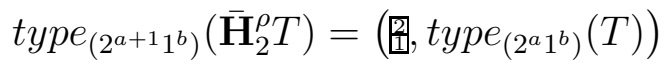

\section{$6 \quad$ Statistics for $\mu=\left(32^{a} 1^{b}\right)$ or $\mu=\left(42^{a} 1^{b}\right)$}

When $H_{m}^{t}$ acts on a Macdonald symmetric function, we observe experimentally that if $m \geq$ $\mu_{1}-1$, then $H_{m}^{t} H_{\mu}[X ; q, t]$ is Schur positive. This can be explained for the case that $\mu=\left(2^{a} 1^{b}\right)$ where we have a combinatorial interpretation for the terms. We then use this result to show that $H^{S} H_{\left(2^{a} 1^{b}\right)}[X ; q, t]$ is Schur positive when $S$ is a standard tableaux of size 3 or 4 where the $H^{S}$ operators are given in equations (3.6) and (4.8).

The definition of $\operatorname{type}_{\left(2^{a+1} 1^{b}\right)}\left(\mathbf{H}_{2}^{\rho} T\right)$ is (피, $\operatorname{type}_{\left(2^{a} 1^{b}\right)}\left(\mathbf{H}_{2}^{-1} \mathbf{H}_{2}^{\rho} T\right)$ ), Proposition 5.10] is

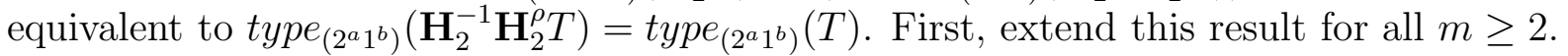

In all of the results in this section we will assume that $m \geq 2$. 
Lemma 6.1 Let $T \in S T^{n}$ and let $\rho$ be a partition of $2 n+m$ such that $\rho / \lambda(T) \in \mathcal{H}_{n+m}$ then

$$
\operatorname{type}_{\left(2^{a} 1^{b}\right)}\left(\mathbf{H}_{m}^{-1} \mathbf{H}_{m}^{\rho} T\right)=\operatorname{type}_{\left(2^{a} 1^{b}\right)}(T)
$$

Similarly, if $\rho / \lambda(T) \in \mathcal{V}_{n+m}$ then

$$
\operatorname{type}_{\left(2^{a} 1^{b}\right)}\left(\mathbf{H}_{m}^{-1} \overline{\mathbf{H}}_{m}^{\rho} T\right)=\operatorname{type}_{\left(2^{a} 1^{b}\right)}(T)
$$

Proof: We will just consider the first case since the proof of the second is mostly a matter of changing $\mathbf{H}_{k}^{\theta}$ to $\overline{\mathbf{H}}_{k}^{\theta}$ and 'row' to 'column.'

Let $\tilde{\rho}=\lambda\left(\mathbf{H}_{m}^{\rho} T\right)^{r}$. We claim that $\mathbf{H}_{2}^{\tilde{\rho}} \mathbf{H}_{m}^{-1} \mathbf{H}_{m}^{\rho} T=\mathbf{H}_{2}^{\rho} T$.

Let $S$ be the tableau of shape $\rho^{r}$ formed by evacuating the cells in $\lambda(T) / \rho^{r}$ and let $R$ be the evacuated cells in the procedures for calculating $\mathbf{H}_{m}^{\rho} T$ and $\mathbf{H}_{2}^{\rho} T$. We will analyze the procedures without making changes in the labels for $\mathbf{H}_{m}^{\rho} T$ and instead of inserting the labels 1 through $m$, insert $m$ 'spaces.' The procedure for calculating the right hand side says row insert two spaces and all of $R$ into $S$.

The procedure for calculating the left hand side says row insert $m$ spaces and all of $R$ into $S$. Next, row evacuate the entire first row of the result, throw away all but 2 of the spaces and reinsert what is left. Clearly, since row insertion and row evacuation are inverses of each other, this is equivalent to the procedure for calculating the right hand side.

The result follows from a couple of applications of Proposition 5.10, since

$$
\begin{aligned}
\operatorname{type}_{\left(2^{a} 1^{b}\right)}\left(\mathbf{H}_{m}^{-1} \mathbf{H}_{m}^{\rho} T\right) & =\operatorname{type}_{\left(2^{a} 1^{b}\right)}\left(\mathbf{H}_{2}^{-1} \mathbf{H}_{2}^{\tilde{\rho}} \mathbf{H}_{m}^{-1} \mathbf{H}_{m}^{\rho} T\right) \\
& =\operatorname{type}_{\left(2^{a} 1^{b}\right)}\left(\mathbf{H}_{2}^{-1} \mathbf{H}_{2}^{\rho} T\right) \\
& =\operatorname{type}_{\left(2^{a} 1^{b}\right)}(T)
\end{aligned}
$$

○

Define an involution, $I_{\lambda}^{n}$, on partitions. Let $\left.\gamma=\rho\right\rfloor_{n}$. If $\lambda_{h}>\gamma_{h}$ then define $I_{\lambda}^{n}(\rho)=\gamma$ with an $n-$ snake of height $h+1$ added. If $\lambda_{h} \leq \gamma_{h}$ then let $I_{\lambda}^{n}(\rho)=\gamma$ with an $n-$ snake of height $h-1$ added. Lemma 3.15 of [22] was the following result.

Lemma $6.2 I_{\lambda}^{n}$ is an involution on partitions $\rho$ such that $\left.\rho / \lambda \in \mathcal{H}_{n}, \rho\right\rfloor_{n}$ exists and $\left.\lambda \neq \rho\right\rfloor_{n}$ with the property that $h t_{n}\left(I_{\lambda}^{n}(\rho)\right)=h t_{n}(\rho) \pm 1$ and $\left.\left.\rho\right\rfloor_{n}=I_{\lambda}^{n}(\rho)\right\rfloor_{n}$.

Example 6.3 Let $n=10, \lambda=(5,5,2)$, and $\rho=(12,5,5)$, then $\rho\rfloor_{n}=(4,4,4)$ and $h t_{n}(\rho)=$ 3. $I_{\lambda}^{n}(\rho)=(13,5,4)$ because $I_{\lambda}^{n}(\rho)=(4,4,4)$ with a 10 snake of height 2 added. 
Lemma 6.4 For a pair $(T, \rho)$ for $T$ a standard tableau, $\rho / \lambda(T) \in \mathcal{H}_{n+m}$

$$
c\left(\mathbf{H}_{m}^{\rho} T\right)=c(T)+\left|\lambda(T) / \rho^{r}\right|+\left(\begin{array}{l}
m \\
2
\end{array}\right)+(m-1) n
$$

Proof: $\quad$ We note that $\mathbf{H}_{m}^{\rho} T=\left(\tilde{T} \uparrow_{m}\right) 12 \cdots m\left(R \uparrow_{m}\right)$ where $\tilde{T}$ and $R$ are given in the procedure for the calculation of $\mathbf{H}_{m}^{\rho} T . T=R \tilde{T}$ and $|R|=\left|\lambda(T) / \rho^{r}\right|$. Apply Fact $5.4 m$ times. $\circ$

Lemma 6.5 The following two conditions are equivalent for a pair $(T, \rho)$ such that $\rho / \lambda(T) \in$ $\mathcal{H}_{n+m}$ and $\left.\rho\right\rfloor_{n}$ exists.

$$
\begin{aligned}
\mathbf{H}_{m}^{-1} \mathbf{H}_{m}^{\rho} T & =T \\
\lambda\left(\mathbf{H}_{m}^{\rho} T\right) & =\rho\rfloor_{n}
\end{aligned}
$$

Moreover, if $(T, \rho)$ satisfies these these conditions, then $h t_{n}(\rho)=1$.

Proof: $\quad$ Since $\rho / \lambda\left(\mathbf{H}_{m}^{\rho} T\right) \in \mathcal{H}_{n}$ then if $\left.\lambda\left(\mathbf{H}_{m}^{\rho} T\right)=\rho\right\rfloor_{n}$ then we have that $\left.\rho / \rho\right\rfloor_{n} \in \mathcal{H}_{n}$, and hence $n=1$.

Let $\tilde{T}$ and $R$ be (respectively) the tableau of shape $\rho^{r}$ and the row of size $\left|\lambda(T) / \rho^{r}\right|$ in the procedure for $\mathbf{H}_{m}^{\rho} T$.

If $\left.\lambda\left(\mathbf{H}_{m}^{\rho} T\right)=\rho\right\rfloor_{n}$ then $\left.\rho\right\rfloor_{n}=\left(\rho_{1}-n, \rho^{r}\right)=\left(m+|R|, \rho^{r}\right)$ hence the first row of $\mathbf{H}_{m}^{\rho} T$ (which must contain $12 \cdots m\left(R \uparrow_{m}\right)$ since it was row inserted in increasing order) is $12 \cdots m\left(R \uparrow_{m}\right)$. Therefore $\mathbf{H}_{m}^{-1} \mathbf{H}_{m}^{\rho} T=R \tilde{T}=T$.

If $\mathbf{H}_{m}^{-1} \mathbf{H}_{m}^{\rho} T=T$ then the first row of $\mathbf{H}_{m}^{\rho} T$ will be denoted by $12 \cdots m \bar{R}$ and the remainder of the tableau will be called $\bar{T}$. We have that $\bar{R} \bar{T}=T$ and we want to show that $|\bar{R}|=|R|$. By Lemma 6.4 and repeated application of Fact 5.4 we have that

$$
c(T)=c(\bar{T} 12 \cdots m \bar{R})-|R|-\left(\begin{array}{c}
m \\
2
\end{array}\right)-(m-1) n=c(T)+|\bar{R}|-|R|
$$

Therefore $\left.\lambda\left(\mathbf{H}_{m}^{\rho} T\right)=\left(m+|R|, \rho^{r}\right)=\rho\right\rfloor_{n} . \quad \circ$

Call a pair that satisfies the conditions of Lemma 6.5 stable and a pair $(T, \rho)$ such that $\rho / \lambda(T) \in \mathcal{H}_{n+m}$ and $\left.\rho\right\rfloor_{n}$ exists and that does not satisfy the conditions of the lemma, unstable. A pair $(T, \rho)$ such that $\rho / \lambda(T) \in \mathcal{H}_{n+m}$ and $\left.\rho\right\rfloor_{n}$ does not exist will be called immaterial. 
Lemma 6.6 There exists an involution $\mathcal{I}^{n, m}$ on pairs $(T, \rho)$ that are unstable. The corresponding pair $(\hat{T}, \tilde{\rho})=\mathcal{I}^{n, m}(T, \rho)$ has the property that $\mathbf{H}_{m}^{\tilde{\rho}} \hat{T}=\mathbf{H}_{m}^{\rho} T, h t_{n}(\tilde{\rho})=h t_{n}(\rho) \pm 1$

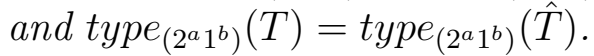

Proof: Begin by setting $\lambda=\lambda\left(\mathbf{H}_{m}^{\rho} T\right)$ and $\tilde{\rho}=I_{\lambda}^{n}(\rho)$ then the property that $h t_{n}(\tilde{\rho})=$ $h t_{n}(\rho) \pm 1$ follows from Lemma 6.2. We want to define $\hat{T}=\left(\mathbf{H}_{m}^{\tilde{\rho}}\right)^{-1} \mathbf{H}_{m}^{\rho} T$, but it is not always possible to apply $\left(\mathbf{H}_{m}^{\tilde{\rho}}\right)^{-1}$ to a tableau. The first $m$ columns of $\rho$ and $\tilde{\rho}$ must be the same because $\rho_{1}$ and $\tilde{\rho}_{1}$ are both at least $n+m$ and the leftmost $m$ columns of $\left.\left.\rho\right\rfloor_{n}=\tilde{\rho}\right\rfloor_{n}$ are the same as the leftmost $m$ columns of both $\rho$ and $\tilde{\rho}$. Therefore the procedure for applying $\left(\mathbf{H}_{m}^{\tilde{\rho}}\right)^{-1}$ to the tableau $\mathbf{H}_{m}^{\rho} T$ evacuates the labels $12 \cdots m$ and hence there is no problem defining $\hat{T}$ in this way.

This is an involution since $I_{\lambda}^{n}(\tilde{\rho})=\rho$ and $T=\left(\mathbf{H}_{m}^{\rho}\right)^{-1} \mathbf{H}_{m}^{\tilde{\rho}} \hat{T}$.

We also have that $\mathbf{H}_{m}^{\tilde{\rho}} \hat{T}=\mathbf{H}_{m}^{\rho} T$, and hence by Lemma 6.1

$$
\begin{aligned}
& \operatorname{type}_{\left(2^{a} 1^{b}\right)}(T)=\operatorname{type}_{\left(2^{a} 1^{b}\right)}\left(\mathbf{H}_{m}^{-1} \mathbf{H}_{m}^{\rho} T\right)=\operatorname{type}_{\left(2^{a} 1^{b}\right)}\left(\mathbf{H}_{m}^{-1} \mathbf{H}_{m}^{\tilde{\rho}} \hat{T}\right)=\operatorname{type}_{\left(2^{a} 1^{b}\right)}(\hat{T}) \\
& \circ
\end{aligned}
$$

Example 6.7 Let $m=2$ and $T=$\begin{tabular}{|l|l|l|}
\hline 5 & \\
\hline 4 & \multicolumn{3}{|c}{ and $\rho=(8,3,1)$} \\
\hline 1 & 2 & 3 \\
\hline
\end{tabular}

We calculate that $\mathbf{H}_{2}^{\rho} T=$\begin{tabular}{|l|l|l|l|}
\hline 6 & & & \\
\hline 3 & 4 & &
\end{tabular} and find that $(T, \rho)$ is an unstable pair. $\tilde{\rho}=$

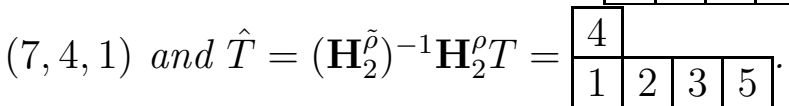

Notice that the types of $T, \hat{T}$, and $\mathbf{H}_{2}^{-1} \mathbf{H}_{2}^{\rho} T$ are all the same. $\operatorname{type}_{(2,2,1)}(T)=$ $\operatorname{type}_{(2,2,1)}(\hat{T})=\operatorname{type}_{(2,2,1)}\left(\mathbf{H}_{2}^{-1} \mathbf{H}_{2}^{\rho} T\right)=($ 띠, 띠, 四).

\section{Proposition 6.8}

$$
H_{m}^{t} H_{\left(2^{a} 1^{b}\right)}[X ; q, t]=\sum_{T} q^{b_{\left(m 2^{a} 1^{b}\right)}^{(T)}} t^{a_{\left(m 2^{a} 1^{b}\right)}(T)} s_{\lambda(T)}[X]
$$


where the sum is over standard tableau $T$ of size $2 a+b+m$ that contain 112$\rceil m$ as a subtableau and $b_{\left(m 2^{a} 1^{b}\right)}(T)=b_{\left(2^{a} 1^{b}\right)}\left(\mathbf{H}_{m}^{-1} T\right)$ and

$$
\begin{aligned}
a_{\left(m 2^{a} 1^{b}\right)}(T)= & c(T)-\left(\begin{array}{c}
m \\
2
\end{array}\right)-(m-1)(2 a+b) \\
& -\sum_{i=1}^{a}((2 a+b+1)-2 i) \chi\left(\operatorname{type}_{\left(2^{a} 1^{b}\right)}\left(\mathbf{H}_{m}^{-1} T\right)_{i}=\right.\text { 띤) }
\end{aligned}
$$

Remark: For the case that $m=1, H_{1}^{t} H_{\left(2^{a} 1^{b}\right)}[X ; q, t]$ is also Schur positive, but not because any of the lemmas in this section hold true and so the following proof does not apply. There is an algebraic explanation for this fact. By using commutation relations (3.1) and (3.3) and Theorem 1.2 we have that $H_{1}^{t} H_{\left(2^{a} 1^{b}\right)}[X ; q, t]=t^{a} H_{\left(2^{a} 1^{b+1}\right)}\left[X ; q t^{-1}, t\right]$.

Proof: Set $n=2 a+b$. For $m \geq 0$, the action of $H_{m}^{t}$ on the Schur function basis is given in Proposition 1.1 and using equation (1.5) , we have that

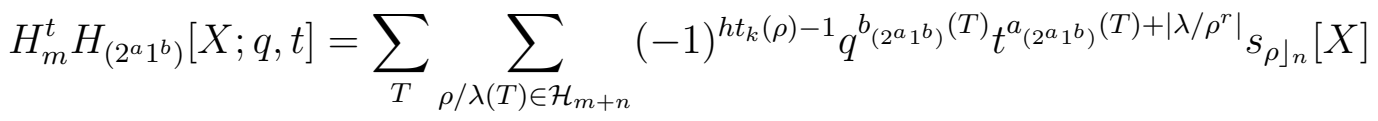

There is exactly one term in equation (6.7) for every pair $(T, \rho)$ where $T$ is a standard tableau of size $n$ and $\rho$ is a partition such that $\rho / \lambda(T) \in \mathcal{H}_{m+n}$ (exactly the pairs for which $\mathbf{H}_{m}^{\rho} T$ is defined). The weight of a pair $(T, \rho)$ will be $(-1)^{h t_{k}(\rho)-1} q^{b\left(2^{\left.a^{b}\right)}(T)\right.} t^{a_{\left(2^{a} 1^{b}\right)}(T)+\left|\lambda / \rho^{r}\right|} s_{\rho\rfloor_{n}}[X]$. Note that the immaterial pairs have weight zero. We will use the lemmas above to show that the unstable pairs cancel and we will be left with the stable pairs which are in bijection with the standard tableau containing $1 / 2 \mid \mathrm{m}$ as a subtableau.

If $(T, \rho)$ is an unstable pair then let $(\hat{T}, \tilde{\rho})=\mathcal{I}^{m, n}(T, \rho)$. Since the $\left(2^{a} 1^{b}\right)-$ types are 
the same then we have that $b_{\left(2^{a} 1^{b}\right)}(T)=b_{\left(2^{a} 1^{b}\right)}(\hat{T})$ and by Proposition 5.9 and Lemma 6.4.

$$
\begin{aligned}
& a_{\left(2^{a} 1^{b}\right)}(T)+\left|\lambda(T) / \rho^{r}\right|=c(T)+\left|\lambda(T) / \rho^{r}\right| \\
& -\sum_{i=1}^{a}((n+1)-2 i) \chi\left(\operatorname{type}_{\left(2^{a} 1^{b}\right)}(T)_{i}=\right.\text { 띠) } \\
& =c\left(\mathbf{H}_{m}^{\rho} T\right)-\left(\begin{array}{l}
m \\
2
\end{array}\right)-(m-1) n \\
& -\sum_{i=1}^{a}((n+1)-2 i) \chi\left(\operatorname{type}_{\left(2^{a} 1^{b}\right)}(T)_{i}=\right.\text { 피) } \\
& =c\left(\mathbf{H}_{m}^{\tilde{\rho}} \hat{T}\right)-\left(\begin{array}{l}
m \\
2
\end{array}\right)-(m-1) n \\
& -\sum_{i=1}^{a}((n+1)-2 i) \chi\left(\operatorname{type}_{\left(2^{a} 1^{b}\right)}(\hat{T})_{i}=\right.\text { 띠) } \\
& =c(\hat{T})+\left|\lambda(\hat{T}) / \tilde{\rho}^{r}\right| \\
& -\sum_{i=1}^{a}((n+1)-2 i) \chi\left(\operatorname{type}_{\left(2^{a} 1^{b}\right)}(\hat{T})_{i}=\right.\text { 띠) } \\
& =a_{\left(2^{a} 1^{b}\right)}(\hat{T})+\left|\lambda(\hat{T}) / \tilde{\rho}^{r}\right|
\end{aligned}
$$

Therefore the unstable pairs $(T, \rho)$ and $(\hat{T}, \tilde{\rho})$ have the same weight but opposite sign.

If $(T, \rho)$ is a stable pair then by Lemma 6.5 the sign of the term is positive and hence are the only terms that contribute to this sum. The map that sends $(T, \rho)$ to $\mathbf{H}_{m}^{\rho} T$ is a bijection from these pairs to the standard tableaux that contain $112 / 2 m$ as a subtableau. Also remark that by Proposition 5.9 and Lemma 6.4 for a tableau of this sort

$$
\begin{aligned}
& a_{\left(2^{a} 1^{b}\right)}\left(\mathbf{H}_{m}^{-1} T\right)+\left|\lambda(T) / \rho^{r}\right|=c\left(\mathbf{H}_{m}^{-1} T\right)+\left|\lambda(T) / \rho^{r}\right| \\
& -\sum_{i=1}^{a}((n+1)-2 i) \chi\left(\operatorname{type}_{\left(2^{a} 1^{b}\right)}\left(\mathbf{H}_{m}^{-1} T\right)_{i}=\text { 띨 }\right) \\
& =c(T)-\left(\begin{array}{c}
m \\
2
\end{array}\right)-(m-1) n \\
& -\sum_{i=1}^{a}((n+1)-2 i) \chi\left(\operatorname{type}_{\left(2^{a} 1^{b}\right)}\left(\mathbf{H}_{m}^{-1} T\right)_{i}=\text { 띠니 }\right)
\end{aligned}
$$

Hence, the exponent of $t$ in the weight of the pair agrees with our definition of $a_{\left(m 2^{a} 1^{b}\right)}(T)$. ○ 


\section{Proposition 6.9}

$$
\bar{H}_{m}^{t} H_{\left(2^{a} 1^{b}\right)}[X ; q, t]=\sum_{T} q^{b_{\left(m 2^{a} 1^{b}\right)}(T)-\left(\begin{array}{c}
m \\
2
\end{array}\right) t^{a_{\left(m 2^{a} 1^{b}\right)}(T)}} s_{\lambda(T)}[X]
$$

where the sum is over standard tableau $T$ of size $2 a+b+m$ that contain $\frac{m}{2}$ as a subtableau and for these tableaux, $b_{\left(m 2^{a} 1^{b}\right)}(T)=b_{\left(2^{a} 1^{b}\right)}\left(\mathbf{H}_{m}^{-1} T\right)+\left(\begin{array}{c}m \\ 2\end{array}\right)$ and

$$
a_{\left(m 2^{a} 1^{b}\right)}(T)=c(T)-\sum_{i=1}^{a}((2 a+b+1)-2 i) \chi\left(\operatorname{type}_{\left(2^{a} 1^{b}\right)}\left(\mathbf{H}_{m}^{-1} T\right)_{i}=\text { 四 }\right)
$$

Proof: All of the arguments and lemmas in this section can be transposed and traced through for the case of $\bar{H}_{m}^{t}$ and $\overline{\mathbf{H}}_{m}^{\rho}$, but an algebraic method is much easier. Set $n=2 a+b$. We have that $H_{\mu}[X ; q, t]=\omega t^{n(\mu)} q^{n\left(\mu^{\prime}\right)} H_{\mu}[X ; 1 / q, 1 / t]$ where $n(\mu)=\sum_{i}(i-1) \mu_{i}$ by [19, eq. 8.14, p.354], and hence

$$
\begin{aligned}
\bar{H}_{m}^{t} H_{\left(2^{a} 1^{b}\right)}[X ; q, t] & =\omega H_{m}^{1 / t} \omega R^{t} H_{\left(2^{a} 1^{b}\right)}[X ; q, t] \\
& =\omega H_{m}^{1 / t} t^{n} t^{n\left(2^{a} 1^{b}\right)} q^{n(a+b, a)} H_{\left(2^{a} 1^{b}\right)}[X ; 1 / q, 1 / t] \\
& =t^{n+n\left(2^{a} 1^{b}\right)} q^{a} \sum_{T} t^{-a\left(m 2^{a} 1^{b}\right)}{ }^{(\omega T)} q^{-b_{\left(m 2^{a} 1^{b}\right)}(\omega T)} s_{\lambda(T)}[X] \\
& =\sum_{T} t^{n+n\left(2^{a} 1^{b}\right)-a_{\left(m 2^{a} 1^{b}\right)}^{(\omega T)}} q^{b_{\left(m 2^{a} 1^{b}\right)}(T)-\left(\begin{array}{c}
m \\
2
\end{array}\right)} s_{\lambda(T)}[X]
\end{aligned}
$$

where the sum here is over all standard tableaux of size $n+m$ that contain the subtableau $m$

2 .

The $t$ exponent can be reduced after this using Proposition 6.8. Fact 5.5, and the 
relation $a_{\left(2^{a} 1^{b}\right)}(\omega T)=n\left(2^{a} 1^{b}\right)-a_{\left(2^{a} 1^{b}\right)}(T)$.

$$
\begin{aligned}
& n+n\left(2^{a} 1^{b}\right)-a_{\left(m 2^{a} 1^{b}\right)}(\omega T)=n\left(2^{a} 1^{b}\right)-c(\omega T)+\left(\begin{array}{l}
m \\
2
\end{array}\right)+m n \\
& +\sum_{i=1}^{a}((n+1)-2 i) \chi\left(\operatorname{type}_{\left(2^{a} 1^{b}\right)}\left(\omega \mathbf{H}_{m}^{-1} T\right)_{i}=\right.\text { Ш2ح) } \\
& =c(T)-\left(\begin{array}{l}
n \\
2
\end{array}\right)+n\left(2^{a} 1^{b}\right) \\
& +\sum_{i=1}^{a}((n+1)-2 i) \chi\left(\operatorname{type}_{\left(2^{a} 1^{b}\right)}\left(\omega \mathbf{H}_{m}^{-1} T\right)_{i}=\right.\text { Ш2ح) } \\
& =c(T)-\sum_{i=1}^{a}((n+1)-2 i) \chi\left(\operatorname{type}_{\left(2^{a} 1^{b}\right)}\left(\mathbf{H}_{m}^{-1} T\right)_{i}=\right.\text { 띠) }
\end{aligned}
$$

$\circ$

To extend these results to $H^{\text {通 }}$ and $H^{\left[H_{2}\right.}$, recovering the statistics involves deleting the first cell and then reducing to the two column case. We introduce the notation of the operator $K_{h}$ that acts on standard tableaux with more than $h$ cells. The result $K_{h} T$ is the tableau formed by deleting the cells with labels 1 through $h$ and then lowering the labels in the remaining tableau by $h$.

\section{Proposition 6.10}

$$
H^{\text {通3 }} H_{\left(2^{a} 1^{b}\right)}[X ; q, t]=\sum_{T} q^{b_{\left(32^{a} 1^{b}\right)}(T)-1} t^{a_{\left(32^{a} 1^{b}\right)}(T)} s_{\lambda(T)}[X]
$$

where the sum is over standard tableau $T$ of size $2 a+b+3$ that contain II $_{1}$ as a subtableau. For these tableaux set $b_{\left(32^{a} 1^{b}\right)}(T)=b_{\left(2^{a} 1^{b}\right)}\left(\mathbf{H}_{2}^{-1} K_{1} T\right)+1$ and

$$
a_{\left(32^{a} 1^{b}\right)}(T)=c(T)-1-(2 a+b)-\sum_{i=1}^{a}((2 a+b+1)-2 i) \chi\left(\operatorname{type}_{\left(2^{a} 1^{b}\right)}\left(\mathbf{H}_{2}^{-1} K_{1} T\right)_{i}=\text { 凹 }\right)
$$

\section{Proof:}

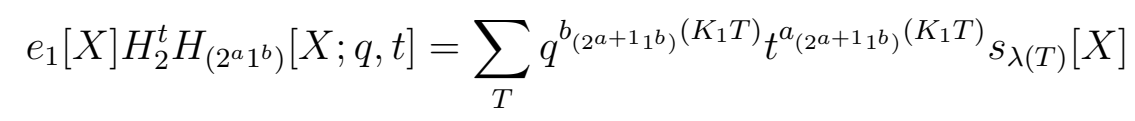




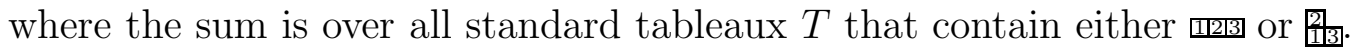

For the tableaux that contain 핀, $c(T)=c\left(K_{1} T\right)+2 a+b+2$ and

$$
\begin{aligned}
a_{\left(2^{a+1} 1^{b}\right)}\left(K_{1} T\right)= & c\left(K_{1} T\right)-\sum_{i=1}^{a+1}((2 a+b+3)-2 i) \chi\left(\operatorname{type}_{\left(2^{a+1} 1^{b}\right)}\left(K_{1} T\right)_{i}=\text { 띠 }\right) \\
= & c(T)-(2 a+b+2)-(2 a+b+1) \\
& \quad-\sum_{i=1}^{a+1}((2 a+b+1)-2 i) \chi\left(\operatorname{type}_{\left(2^{a} 1^{b}\right)}\left(\mathbf{H}_{2}^{-1} K_{1} T\right)_{i}=\right.\text { 띤) }
\end{aligned}
$$

For those tableau that have $\mathbf{H}_{3}^{-1} T=\mathbf{H}_{2}^{-1} K_{1} T$, it is clear that equation (6.24) is equal to $a_{\left(32^{a} 1^{b}\right)}(T)$. For the rest we need verify that $\operatorname{type}_{\left(2^{a} 1^{b}\right)}\left(\mathbf{H}_{2}^{-1} K_{1} T\right)=$ type $_{\left(2^{a} 1^{b}\right)}\left(\mathbf{H}_{3}^{-1} T\right)$.

Let $\bar{T}=\mathbf{H}_{3}^{-1} T$ so that $T=\mathbf{H}_{3}^{\left(3+2 a+b+\lambda(T)_{1}, \lambda(T)^{r}\right)} \bar{T}$. Therefore we have that $K_{1} T=$ $K_{1} \mathbf{H}_{3}^{\left(3+2 a+b+\lambda(T)_{1}, \lambda(T)^{r}\right)} \bar{T}=\mathbf{H}_{2}^{\left(2+2 a+b+\lambda(T)_{1}, \lambda(T)^{r}\right)} \bar{T}$.

$$
\operatorname{type}_{\left(2^{a} 1^{b}\right)}\left(\mathbf{H}_{2}^{-1} K_{1} T\right)=\operatorname{type}_{\left(2^{a} 1^{b}\right)}\left(\mathbf{H}_{2}^{-1} \mathbf{H}_{2}^{\left(2+2 a+b+\lambda(T)_{1}, \lambda(T)^{r}\right)} \bar{T}\right)=\operatorname{type}_{\left(2^{a} 1^{b}\right)}(\bar{T})
$$

From this we can conclude that

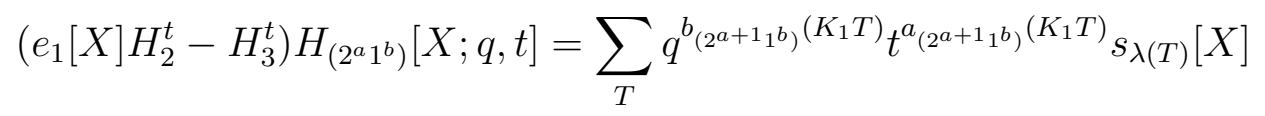

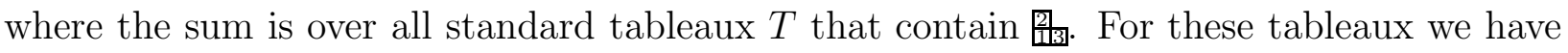
that $c(T)=c\left(K_{1} T\right)$ and hence

$$
\begin{aligned}
& a_{\left(2^{a+1} 1^{b}\right)}\left(K_{1} T\right)=c\left(K_{1} T\right)-\sum_{i=1}^{a+1}((2 a+b+3)-2 i) \chi\left(\operatorname{type}_{\left(2^{a+1} 1^{b}\right)}\left(K_{1} T\right)_{i}=\text { 밀 }\right) \\
& =c(T)-(2 a+b+1)-\sum_{i=1}^{a}((2 a+b+1)-2 i) \chi\left(\operatorname{type}_{\left(2^{a} 1^{b}\right)}\left(\mathbf{H}_{2}^{-1} K_{1} T\right)_{i}=\text { 띠 }\right)
\end{aligned}
$$

○

A similar proof verifies that the operator $H^{[]_{2}}$ works as expected.

\section{Proposition 6.11}

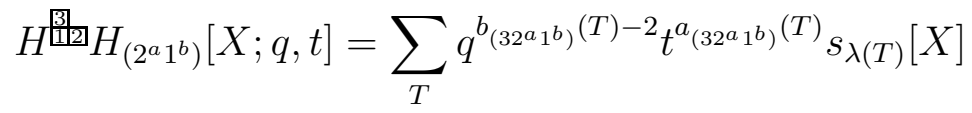




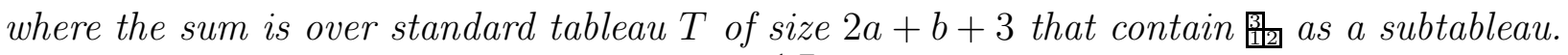
For these tableaux set $b_{\left(32^{a} 1^{b}\right)}(T)=b_{\left(2^{a} 1^{b}\right)}\left(\mathbf{H}_{2}^{-1} \bar{T}\right)+2$ and

$$
a_{\left(32^{a} 1^{b}\right)}(T)=c(T)-2-(2 a+b)-\sum_{i=1}^{a}((2 a+b+1)-2 i) \chi\left(\operatorname{type}_{\left(2^{a} 1^{b}\right)}\left(\mathbf{H}_{2}^{-1} K_{1} T\right)_{i}=\right.\text { 四) }
$$

These propositions together as well as Theorem [1.3 and equation (3.7) show the following theorem.

\section{Theorem 6.12}

$$
H_{\left(32^{a} 1^{b}\right)}[X ; q, t]=\sum_{T} q^{b_{\left(32^{a} 1^{b}\right)}(T)} t^{a_{\left(32^{a} 1^{b}\right)}(T)} s_{\lambda(T)}[X]
$$

where the sum is over all standard tableaux $T$ of size $3+2 a+b$ and $a_{\left(32^{a} 1^{b}\right)}(T)$ and $b_{\left(32^{a} 1^{b}\right)}(T)$ are defined in Propositions 6.8, 6.9, 6.10 and 6.11.

Unfortunately, since there is no general formula for the $H^{S}$ (each one is a special case) and each must be checked separately to show that they have the property that $H^{S} H_{\left(2^{a} 1^{b}\right)}[X ; q, t]$ is Schur positive.

For $T$ a standard tableau of size $2 a+b+4$ that contains the standard tableau $S$ of size 4 we will define that

$$
\begin{gathered}
a_{\left(42^{a} 1^{b}\right)}(T)=c(T)-\alpha_{S}-\beta_{S}(2 a+b)-\sum_{i=1}^{a}((2 a+b+1)-2 i) \chi\left(\operatorname{type}_{\left(2^{a} 1^{b}\right)}\left(\theta_{S}(T)\right)_{i}=\right.\text { Ш2) } \\
b_{\left(42^{a} 1^{b}\right)}(T)=b_{\left(2^{a} 1^{b}\right)}\left(\theta_{S}(T)\right)+\gamma_{S}
\end{gathered}
$$

where $\alpha_{S}, \beta_{S}, \theta_{S}(T)$, and $\gamma_{S}$ are given in Table 1 .

The format for the statistics is the same, just a few variables change for each equation. It is easier to give a table of values for the parts of this equation that change than it is to describe a procedure for calculating them. Below is a table for the statistics for $H_{\left(42^{a} 1^{b}\right)}[X ; q, t]$ (The values in the table for $H_{\left(32^{a} 1^{b}\right)}[X ; q, t]$ follow from Propositions 6.8, 6.9. 6.10 and 6.11). 


\begin{tabular}{|c|c|c|c|c|c|}
\hline $\mathrm{S}$ & $\alpha_{S}$ & $\beta_{S}$ & $\theta_{S}(T)$ & $\gamma_{S}$ & $H^{S}$ \\
\hline पायाओप & 6 & 3 & $\mathbf{H}_{4}^{-1} T$ & 0 & $H_{4}^{t}$ \\
\hline$\frac{21}{1314}$ & 3 & 2 & $\mathbf{H}_{3}^{-1} K_{1} T$ & 1 & $e_{1} H_{3}^{t}-H_{4}^{t}$ \\
\hline 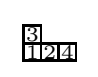 & 4 & 2 & $\mathbf{H}_{2}^{-1} K_{2} T$ & 2 & $h_{2} H_{2}^{t}-H_{4}^{t}$ \\
\hline 贯1213 & 5 & 2 & $\mathbf{H}_{2}^{-1} K_{2} T$ & 3 & $h_{2} \bar{H}_{2}^{t}-h_{1} \bar{H}_{3}^{t}+\bar{H}_{4}^{t}$ \\
\hline 䢁 & 4 & 2 & $\mathbf{H}_{2}^{-1} K_{2} T$ & 2 & $h_{2} H_{2}^{t}-H_{4}^{t}$ \\
\hline 迸 & 2 & 1 & $\mathbf{H}_{2}^{-1} K_{2} T$ & 4 & $e_{2} \bar{H}_{2}^{t}-\bar{H}_{4}^{t}$ \\
\hline 臭 & 1 & 1 & $\mathbf{H}_{2}^{-1} K_{2} T$ & 3 & $e_{2} H_{2}^{t}-e_{1} H_{3}^{t}+H_{4}^{t}$ \\
\hline 圈 & 2 & 1 & $\mathbf{H}_{2}^{-1} K_{2} T$ & 4 & $e_{2} \bar{H}_{2}^{t}-\bar{H}_{4}^{t}$ \\
\hline 圈 & 3 & 1 & $\mathbf{H}_{3}^{-1} K_{1} T$ & 5 & $e_{1} \bar{H}_{3}^{t}-\bar{H}_{4}^{t}$ \\
\hline$\frac{7}{\frac{3}{1}}$ & 0 & 0 & $\mathbf{H}_{4}^{-1} T$ & 6 & $\bar{H}_{4}^{t}$ \\
\hline 파] & 3 & 2 & $\mathbf{H}_{3}^{-1} T$ & 0 & $H_{3}^{t}$ \\
\hline 通3 & 2 & 1 & $\mathbf{H}_{2}^{-1} K_{1} T$ & 1 & $e_{1} H_{2}^{t}-H_{3}^{t}$ \\
\hline 國 & 1 & 1 & $\mathbf{H}_{2}^{-1} K_{1} T$ & 2 & $e_{1} \bar{H}_{2}^{t}-\bar{H}_{3}^{t}$ \\
\hline$\frac{3}{1}$ & 0 & 0 & $\mathbf{H}_{3}^{-1} T$ & 3 & $\bar{H}_{3}^{t}$ \\
\hline
\end{tabular}

Table 1: Values for variable pieces of formulas (6.29) and 6.30) 


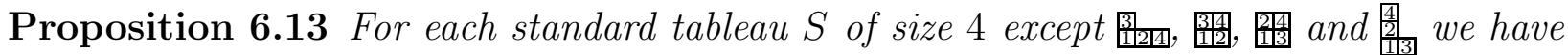
that

$$
H^{S} H_{\left(2^{a} 1^{b}\right)}[X ; q, t]=\sum_{T} q^{b}{ }_{\left(42^{a} 1^{b}\right)}^{(T)-\gamma_{S}} t^{a_{\left(42^{a} 1^{b}\right)}}{ }^{(T)} s_{\lambda(T)}[X]
$$

where the sum is over all standard tableaux $T$ of size $4+2 a+b$ that contain $S$ as a subtableau. In addition we have that

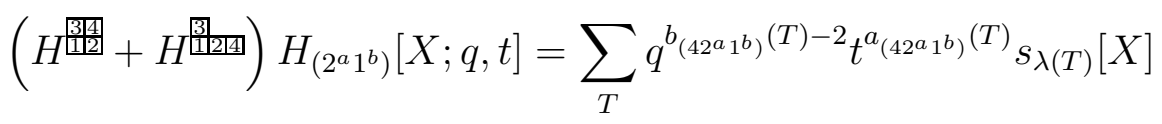

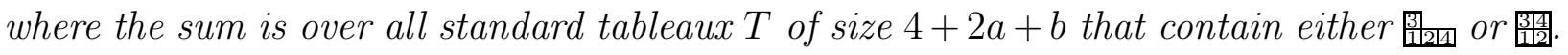
Furthermore,

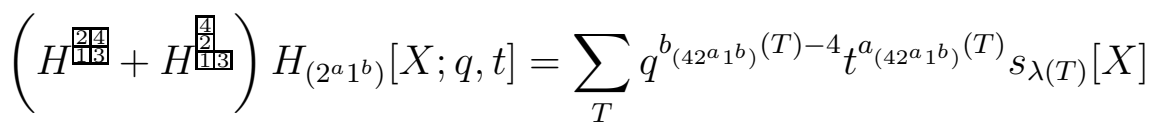

where the sum is over all standard tableaux $T$ of size $4+2 a+b$ that contain either 寈 or 圈.

The fact that $H_{\left(42^{a} 1^{b}\right)}[X ; q, t]$ is Schur positive follows immediately from this proposition and Theorem 1.4 and equation (4.9).

\section{Theorem 6.14}

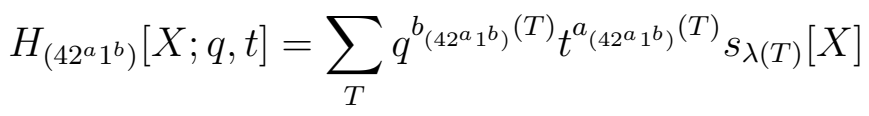

where the sum is over all standard tableaux $T$ of size $4+2 a+b$.

Proof: $\quad$ (of Proposition 6.13)

We note that if $T$ contains 피나 or $\frac{1}{2}$, it is not necessarily the case that $\mathbf{H}_{4}^{-1} T=$ $\mathbf{H}_{3}^{-1} K_{1} T=\mathbf{H}_{2}^{-1} K_{2} T$, but it will be true that $\operatorname{type}_{\left(2^{a} 1^{b}\right)}\left(\mathbf{H}_{4}^{-1} T\right)=\operatorname{type}_{\left(2^{a} 1^{b}\right)}\left(\mathbf{H}_{3}^{-1} K_{1} T\right)=$

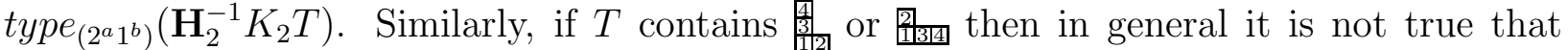
$\mathbf{H}_{3}^{-1} K_{1} T=\mathbf{H}_{2}^{-1} K_{2} T$, but it will be true that $\operatorname{type}_{\left(2^{a} 1^{b}\right)}\left(\mathbf{H}_{3}^{-1} K_{1} T\right)=\operatorname{type}_{\left(2^{a} 1^{b}\right)}\left(\mathbf{H}_{2}^{-1} K_{2} T\right)$. This follows from a similar argument to the one used in equation (6.25).

For each operator $H^{S}$ we must verify that it has the property stated in the propostion. The case of $H^{\text {[121314 }}$ and $H^{2}$ follow from Proposition 6.8 and 6.9 respectively. 
$H^{[1] 3140:}$

Since $H_{3}^{t} H_{\left(2^{a} 1^{b}\right)}[X ; q, t]$ is a generating function for the standard tableaux that contain एथ23 then $e_{1}[X] H_{3}^{t} H_{\left(2^{a} 1^{b}\right)}[X ; q, t]$ is a generating function that for the standard tableaux that

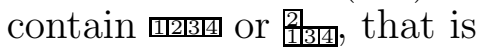

$$
e_{1}[X] H_{3}^{t} H_{\left(2^{a} 1^{b}\right)}[X ; q, t]=\sum_{T} q^{b\left(2^{a} 1^{b}\right)}\left(\mathbf{H}_{3}^{-1} K_{1} T\right) t^{a_{\left(32^{a} 1^{b}\right)}\left(K_{1} T\right)} s_{\lambda(T)}[X]
$$

To verify this proposition in this case we need to show that $a_{\left(32^{a} 1^{b}\right)}\left(K_{1} T\right)=a_{\left(42^{a} 1^{b}\right)}(T)$ for these tableaux. For the tableaux that contain ${ }_{131,4}$, this is true by definition. For the tableaux that contain 핌 we note that $c\left(K_{1} T\right)=c(T)-(2 a+b+3)$ and $\operatorname{type}_{\left(2^{a} 1^{b}\right)}\left(\mathbf{H}_{4}^{-1} T\right)=$ $\operatorname{type}_{\left(2^{a} 1^{b}\right)}\left(\mathbf{H}_{3}^{-1} K_{1} T\right)$.

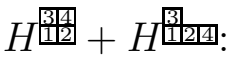

$h_{2} H_{2}^{t} H_{\left(2^{a} 1^{b}\right)}[X ; q, t]$ is a generating function for the standard tableaux that contain

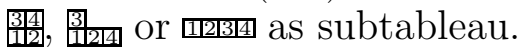

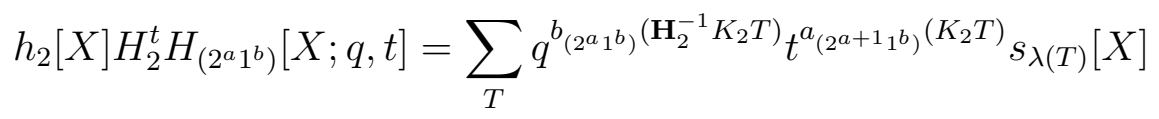

We verify for these standard tableaux that $a_{\left(2^{a+1} 1^{b}\right)}\left(K_{2} T\right)=a_{\left(42^{a} 1^{b}\right)}(T)$ For the tableaux

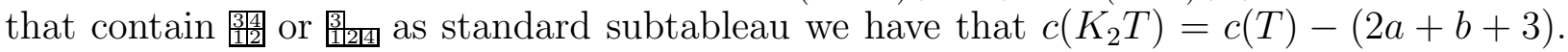
For the tableaux that contain 띠피, $c\left(K_{2} T\right)=c(T)-(2(2 a+b)+5)$ and we note that $\operatorname{type}_{\left(2^{a} 1^{b}\right)}\left(\mathbf{H}_{3}^{-1} K_{1} T\right)=\operatorname{type}_{\left(2^{a} 1^{b}\right)}\left(\mathbf{H}_{2}^{-1} K_{2} T\right)$.

$H^{\text {年禹: }}$

$e_{2} H_{2}^{t} H_{\left(2^{a} 1^{b}\right)}[X ; q, t]$ is a generating function for the standard tableaux that contain 道 and $1 \frac{13 \text { as }}{13}$ as a subtableau with the formula

$$
h_{2}[X] H_{2}^{t} H_{\left(2^{a} 1^{b}\right)}[X ; q, t]=\sum_{T} q^{b_{\left(2^{a} 1^{b}\right)}\left(\mathbf{H}_{2}^{-1} K_{2} T\right)} t^{a_{\left(2^{a+1} 1^{b}\right)}\left(K_{2} T\right)} s_{\lambda(T)}[X]
$$

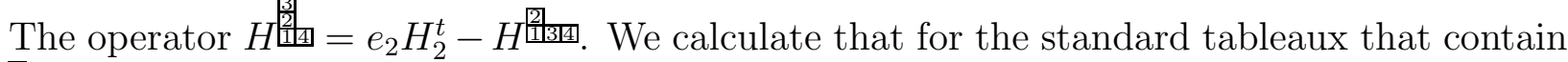
造, $c\left(K_{2} T\right)=c(T)$ and for the standard tableaux the contain 11314, $c\left(K_{2} T\right)=c(T)-(2 a+b+2)$.

The verification of the cases for the operators with the transpose tableaux is nearly identical or an algebraic argument can be used. $\alpha_{S}=6-\alpha_{\omega S}$ and $\beta_{S}=3-\beta_{\omega S}$ for all standard tableaux $S$ of size 4 . Let $n=2 a+b$. It follows from the relation $H^{S}=\left.\omega H^{\omega S}\right|_{t \rightarrow 1 / t} \omega R^{t}$ and equation (6.18) that 


$$
H^{\omega S} H_{\left(2^{a} 1^{b}\right)}[X ; q, t]=\sum_{T} t^{n+n\left(2^{a} 1^{b}\right)-a_{\left(42^{a} 1^{b}\right)}(\omega T)} q^{n(a+b, a)-b_{\left(42^{a} 1^{b}\right)}(\omega T)} s_{\lambda(T)}[X]
$$

where the sum is over all standard tableaux $T$ that contain $\omega S$.

Now for the statistic, we have that

$$
\begin{aligned}
& n+n\left(2^{a} 1^{b}\right)-a_{\left(42^{a} 1^{b}\right)}(\omega T)=n+n\left(2^{a} 1^{b}\right)-\left(c(\omega T)-\alpha_{S}-\beta_{S} n\right. \\
& -\sum_{i=1}^{a}((2 a+b+1)-2 i) \chi\left(\operatorname{type}_{\left(2^{a} 1^{b}\right)}\left(\theta_{S}(\omega T)\right)_{i}=\text { Шح) }\right) \\
& =c(T)-(n+4)(n+3) / 2+n(n-1) / 2+n+\alpha_{S}+\beta_{S} n \\
& -\sum_{i=1}^{a}((2 a+b+1)-2 i) \chi\left(\operatorname{type}_{\left(2^{a} 1^{b}\right)}\left(\theta_{\omega S}(T)\right)_{i}=\text { 피 }\right) \\
& =c(T)-\left(3-\beta_{S}\right) n-\left(6-\alpha_{S}\right) \\
& -\sum_{i=1}^{a}((2 a+b+1)-2 i) \chi\left(\operatorname{type}_{\left(2^{a} 1^{b}\right)}\left(\theta_{\omega S}(T)\right)_{i}=\text { प⿴囗⿰丿㇄口阝 }\right) \\
& =a_{\left(42^{a} 1^{b}\right)}(T)
\end{aligned}
$$

$\circ$

It makes sense here to generalize the notion of the $\operatorname{type}_{\left(2^{a} 1^{b}\right)}$ as it was introduced in 22 to the case of $\left(32^{a} 1^{b}\right)$ and $\left(42^{a} 1^{b}\right)$. For $m=3$ or $4, T$ be a standard tableau of size $m+2 a+b$ that contains the standard tableau $S$ of size $m$. Define the type $_{\left(m 2^{a} 1^{b}\right)}(T)=$ $\left(S\right.$, type $\left._{\left(2^{a} 1^{b}\right)}\left(\theta_{S}(T)\right)\right)$ where $\theta_{S}(T)$ is given in Table 1 .

We finish this article by noting that the following analog to Conjecture 4.8 of [22] seems to be true by experimental calculation.

Conjecture 6.15 The number of tableaux of a fixed $\left(m 2^{a} 1^{b}\right)$-type, $(S, s)$, and fixed $a_{\left(m 2^{a} 1^{b}\right)}$ value, $i$, increases to a maximum and then decreases as $i$ ranges over all possible values.

More precisely stated, if $S$ is a standard tableau of size $m$ ( $m=3$ or 4$)$ and $s$ is a sequence of a standard tableaux of size 2 and $b$ of size 1 , then let $A_{(S, s)}^{i}=\#\{T \mid T \in$ $\left.S T^{m+2 a+b}, \operatorname{type}_{\left(m 2^{a} 1^{b}\right)}(T)=(S, s), a_{\left(m 2^{a} 1^{b}\right)}(T)=i\right\}$. The sequence $A_{(S, s)}^{*}=\left(A_{(S, s)}^{0}, A_{(S, s)}^{1}, A_{(S, s)}^{2}, \ldots\right)$ is unimodal. 
Example 6.16 Let $s=(\square, \square, \square)$ and let $S$ range over all standard tableaux of size 3 .

$$
\begin{aligned}
& S=\text { 따의 } \quad A_{(S, s)}^{*}=(1,2,3,4,2,1,1) \\
& S=\text { 通 } \quad A_{(S, s)}^{*}=(2,4,6,5,4,2,1) \\
& S=\text { 鳏 } \quad A_{(S, s)}^{*}=(1,2,4,5,6,4,2) \\
& S=\text { 图 } \quad A_{(S, s)}^{*}=(1,1,2,4,3,2,1)
\end{aligned}
$$

Let $s=(\square, \square)$ and let $S$ range over all standard tableaux of size 4 . This partitions all of the standard tableaux of size 6 into 10 different types.

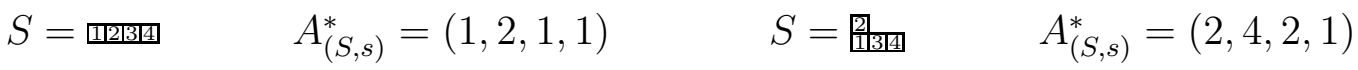

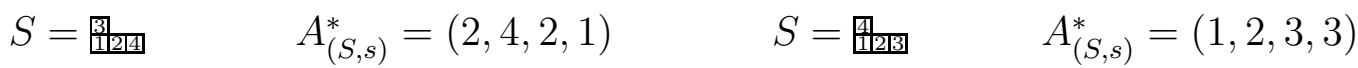

$$
\begin{aligned}
& S=\text { 速 } \quad A_{(S, s)}^{*}=(1,2,2,1) \quad S=\text { 䊚 } \quad A_{(S, s)}^{*}=(1,2,2,1) \\
& S=\text { 竡 } \quad A_{(S, s)}^{*}=(3,3,2,1) \quad S=\text { 通 } \quad A_{(S, s)}^{*}=(1,2,4,2) \\
& S=\text { 通 } \quad A_{(S, s)}^{*}=(1,2,4,2) \quad S=\text { 置 } \quad A_{(S, s)}^{*}=(1,1,2,1)
\end{aligned}
$$

Example 6.17 Let $s=($ प⿴囗十, 四, 四) and $S$ range over all standard tableaux of size 3 .

$$
\begin{aligned}
& S=\text { 판] } \quad A_{(S, s)}^{*}=(1,4,6,8,7,6,4,2,1,1) \\
& S=\text { 还了 } \quad A_{(S, s)}^{*}=(2,3,8,12,13,10,8,4,2,1) \\
& S=\text { 遮 } \quad A_{(S, s)}^{*}=(0,0,2,7,12,14,13,9,4,2) \\
& S=\frac{3}{2} \quad A_{(S, s)}^{*}=(0,0,1,3,5,7,7,4,2,1)
\end{aligned}
$$

\section{References}

[1] L. Butler. Subgroup Lattices and Symmetric Functions, Mem. Amer Math Soc., 112 (1994).

[2] W. Brockman. Nilpotent orbit varieties and the atomic decomposition of the $q$-Kostka polynomials. UCSD Thesis, 1997. 
[3] S. Fishel, Statistics for special q,t-Kostka polynomials, Proc. Amer. Math. Soc., Vol. 123, No. 10, October 1993.

[4] W. Fulton. "Young Tableaux: With Applications to Representation Theory and Geometry," volume 35 of London Mathematical Society Student Texts. Cambridge UP, 1997.

[5] A.M. Garsia, Orthogonality of Milne's polynomials and raising operators, Discrete Mathematics, North-Holland. 99 (1992), 247-264.

[6] A. M. Garsia, M. Haiman, Some natural bigraded $S_{n}$-modules and $q, t$-Kostka coefficients. The Foata Festschrift. Electron. J. Combin. 3 (1996), no. 2, Research Paper 24, approx. $60 \mathrm{pp}$.

[7] A. M. Garsia, J. Remmel, Plethystic Formulas and positivity for q,t-Kostka Coefficients. Mathematical Essays in Honor of Gian-Carlo Rota, B. Sagan \& R. Stanley. Ed., Progress in Mathematics V 161, Birkhauser (1998) pp. 245-262

[8] G.N. Han, Une version géometrique de la construction de Kerov-Kirillov-Reshetikhin, Séminaire Logharingien de Combinatoire, B31a, I.R.M.A. Strasbourg (1994), 71-85.

[9] N. Jing, Vertex operators and Hall-Littlewood symmetric functions, Adv. Math., 87 (1991), 226-248.

[10] A. Kirillov and M. Noumi, Affine Hecke algebras and raising operators for Macdonald polynomials, Duke Math. J., to appear.

[11] A. N. Kirillov and N. Yu. Reshetikhin, Combinatorics, Bethe ansatz, and representations of the symmetric group, Zap. Nauchn. Sem. Leningrad. Otdel Mat. Inst. Steklov. (LOMI) 155 (1986), 50-64; English trans., J. Soviet Math. 41 (1988), 916-924.

[12] A. N. Kirillov and N. Yu. Reshetikhin, The Bethe anstz and the combinatorics of Young tableaux, Zap. Nauch. Sem. Leningrad. Otdel. Mat. Inst. Steklov. (LOMI) 155 (1986) 65-115; English transl., J. Soviet Math. 41 (1988), 925-955.

[13] A. N. Kirillov and M. Shimizono, A generalization of the Kostka Foulkes polynomials, (preprint math.QA/9803062).

[14] A. N. Kirillov, A. Schilling, M. Shimozono, A bijection between Littlewood-Richardson tableaux and rigged configurations, (preprint math.CO/9901037).

[15] L. Lapointe and L. Vinet, A short proof of the integrality of the Macdonald $(q, t)$-Kostka coefficients, Duke Math. J. 91 (1998), 205-214. 
[16] L. Lapointe and J. Morse, Tableaux statistics for two part Macdonald polynomials, (preprint math.CO/9812001)

[17] A. Lascoux, Cyclic permutations on words, tableaux, and harmonic polynomials, Proceedings of the Hyderabad Conference on Algebraic Groups (Hyderabad,1989), pages 323-347, Madras. Manoj Prakashan. (1991)

[18] A. Lascoux and M. P. Schützenberger, Le Monoid plaxique, Noncommutative structures in Algebra and Geometric Combinatorics, CNR, Rome. 109 (1981), 129-156.

[19] I.G. Macdonald, "Symmetric Functions and Hall Polynomials," Oxford Mathematical Monographs, Oxford UP, second edition, 1995.

[20] J. Stembridge, Some particular entries of the two parameter Kostka matrix, Proc. Amer. Math. Soc. 121 (1994), 367-373.

[21] S. Veigneau, ACE, an Algebraic Combinatorics Environment for the computer algebra system MAPLE, User's Reference Manual, Version 3.0, IGM 98-11, Université de Marne-la-Vallée, 1998.

[22] M. A. Zabrocki, A Macdonald Vertex Operator and Standard Tableaux Statistics for the Two-Column $(q, t)$-Kostka Coefficients, Electron. J. Combinat. 5, R45 (1998), 46pp.

[23] M. A. Zabrocki, The Action of the Hall-Littlewood vertex operator, (preprint). 